\title{
Synthesis and Intramolecular Pericyclization of 1-Azulenyl Thioketones
}

Shunji Ito, ${ }^{*} \dagger$ Tetsuo Okujima, ${ }^{\ddagger}$ Shigeru Kikuchi, ${ }^{\ddagger}$ Taku Shoji, ${ }^{\ddagger}$ Noboru Morita,${ }^{\ddagger}$ Toyonobu Asao, ${ }^{\ddagger}$

Tadaaki Ikoma, ${ }^{\S}$ Shozo Tero-Kubota, ${ }^{\perp}$ Jun Kawakami, $^{\dagger}$ and Akio Tajiri ${ }^{\dagger}$

${ }^{\dagger}$ Graduate School of Science and Technology, Hirosaki University, Hirosaki 036-8561, Japan

Department of Chemistry, Graduate School of Science, Tohoku University, Sendai 980-8578, Japan

${ }^{\S}$ Institute of Science and Technology, Niigata University, Niigata 950-2181, Japan, PRESTO, Japan Science and Technology Agency, Kawaguchi 332-0012, Japan

${ }^{\perp}$ Office of Cooperative Research and Development, Tohoku University, Sendai 980-8579, Japan

\section{Supporting Information}

(1) General and experimental details of 1b, 2a-d, 5a, 5b, 8b, 8c, 8d, 9b-d, 11, 12, 13b, 14b, 14c, 16,

$18^{+} \cdot \mathrm{PF}_{6}^{-}$, and $19^{+} \cdot \mathrm{PF}_{6}^{-}$

(2) Cyclic voltammograms of $\mathbf{1 8}^{+} \cdot \mathrm{PF}_{6}^{-}$and $\mathbf{1 9}^{+} \cdot \mathrm{PF}_{6}^{-}$ S-16

(3) Spectroelectrograms of $19^{+} \cdot \mathrm{PF}_{6}^{-}$

(4) ESR measurements of $19^{+} \cdot \mathrm{PF}_{6}^{-}$

(5) B3LYP/6-31G ${ }^{* *}$ density functional calculation of 1-azulenecarbothialdehyde

S-26

(6) Relative stability of azuleno $[8,1-b, c]$ thiophene derivatives

S-28

(7) Copies of ${ }^{1} \mathrm{H}$ and ${ }^{13} \mathrm{C}$ NMR spectra of 1a, 1b, 2a-d, 5a, 5b, 8a, 8c, 8d, 9a-d, 11, 12, 13b, 14b, 14c,

$16,17^{+}, \mathbf{1 8}^{+} \cdot \mathrm{PF}_{6}^{-}$, and $19^{+} \cdot \mathrm{PF}_{6}^{-}$ 


\section{General and experimental details}

General. Melting points were determined on a micro melting point apparatus and are uncorrected. Gel permeation chromatography (GPC) was performed on a TOSOH TSKgel $\mathrm{G} 2000 \mathrm{H}_{6}$ with $\mathrm{CHCl}_{3}$ as an eluent. Column chromatography on silica gel and $\mathrm{Al}_{2} \mathrm{O}_{3}$ was performed using MERCK Silica gel 60 Art. 7734 and MERCK Aluminium oxide 90 Art. 1097.

Voltammetry Measurements. The voltammetry measurements were carried out in acetonitrile containing $\mathrm{Et}_{4} \mathrm{NClO}_{4}(0.1 \mathrm{M})$ as a supporting electrolyte utilizing Pt working and auxiliary electrodes, and a reference electrode formed from $\mathrm{Ag} / \mathrm{AgNO}_{3}(0.01 \mathrm{M})$ in acetonitrile containing $n$ - $\mathrm{Bu}_{4} \mathrm{NClO}_{4}(0.1$ $\mathrm{M})$ at the scan rate of $100 \mathrm{mV} \mathrm{s}^{-1}$. The internal reference $\mathrm{Fc} / \mathrm{Fc}^{+}$discharges at $+0.07 \mathrm{~V}$ under these conditions.

Spectroelectrogram Measurements. Sampe solutions were prepared by dissolving carbocations $\mathbf{1 8}^{+}$ and $\mathbf{1 8}^{+}$in benzonitrile containing $\mathrm{Et}_{4} \mathrm{NClO}_{4}(0.1 \mathrm{M})$ and were degassed through an Ar stream. Spectroelectrogram measurements were carried out using a quarts cell $(1 \times 10 \times 35 \mathrm{~mm})$ equipped with a Pt mesh and a wire as the working and counter electrodes, which were separated by a glass filter. The electrical current was monitored by a microampere meter. Spectroelectrograms were measured on a fiber optic spectrometer at $30 \mathrm{sec}$ or 5 min intervals.

1-Benzoylazulene (5a). ${ }^{5}$ A solution of $\mathrm{POCl}_{3}(1.9 \mathrm{~mL}, 20 \mathrm{mmol})$ in THF $(5 \mathrm{~mL})$ was added at room temperature to a solution of azulene (3a) $(1.28 \mathrm{~g}, 10.0 \mathrm{mmol})$ and $N, N$-dimethylbenzamide (4) (3.00 g, $20.1 \mathrm{mmol})$ in THF $(25 \mathrm{~mL})$. The resulting mixture was refluxed for $4 \mathrm{~h}$. The reaction mixture was poured into water, made alkaline with $1 \mathrm{M} \mathrm{NaOH}$, and then extracted with $\mathrm{CHCl}_{3}$. The organic layer 
was washed with water, dried over $\mathrm{MgSO}_{4}$, and concentrated under reduced pressure. The residue was purified by column chromatography on silica gel with benzene to afford 5a (499 $\mathrm{mg}, 22 \%$ ). reddish purple crystals; mp $116.2-117.1^{\circ} \mathrm{C}$; [lit. $\left.{ }^{5} \mathrm{mp} 117-118^{\circ} \mathrm{C}\right]$; MS $(70 \mathrm{eV}) \mathrm{m} / z 232\left(\mathrm{M}^{+}, 68 \%\right), 155\left(\mathrm{M}^{+}\right.$ - Ph, 100), $127\left(\mathrm{M}^{+}-\mathrm{COPh}, 28\right)$; IR (KBr disk) $v_{\max } 1611(\mathrm{C}=\mathrm{O}), 1406,1393,1277,1237,843 \mathrm{~cm}^{-1}$; UV-vis $\left(\mathrm{CH}_{2} \mathrm{Cl}_{2}\right) \lambda_{\max }, \mathrm{nm}$ (log $\left.\varepsilon\right) 237 \mathrm{sh}$ (4.24), 274 (4.26), 306 sh (4.42), 315 (4.49), 383 (4.06), 392 sh (4.05), $494 \mathrm{sh}$ (2.61), 534 (2.37), $571 \mathrm{sh}$ (2.63), $623 \mathrm{sh}(2.17) ;{ }^{1} \mathrm{H}$ NMR (500 MHz, $\left.\mathrm{CDCl}_{3}\right) \delta=9.74$ $\left(\mathrm{d}, 1 \mathrm{H}, J=9.8 \mathrm{~Hz}, \mathrm{H}_{8}\right), 8.49$ (d, 1H, $\left.J=9.8 \mathrm{~Hz}, \mathrm{H}_{4}\right), 8.07$ (d, $\left.1 \mathrm{H}, J=4.1 \mathrm{~Hz}, \mathrm{H}_{2}\right), 7.84$ (dd, 2H, $J=7.3$, $\left.1.4 \mathrm{~Hz}, \mathrm{H}_{2^{\prime}, 6^{\prime}}\right), 7.84\left(\mathrm{dd}, 1 \mathrm{H}, J=10.4,10.1 \mathrm{~Hz}, \mathrm{H}_{6}\right), 7.62\left(\mathrm{dd}, 1 \mathrm{H}, J=10.1,9.8 \mathrm{~Hz}, \mathrm{H}_{7}\right), 7.55(\mathrm{tt}, 1 \mathrm{H}, J=$ 7.3, $\left.1.4 \mathrm{~Hz}, \mathrm{H}_{4^{\prime}}\right), 7.49\left(\mathrm{dd}, 1 \mathrm{H}, J=10.4,9.8 \mathrm{~Hz}, \mathrm{H}_{5}\right), 7.48\left(\mathrm{dd}, 2 \mathrm{H}, J=7.3,7.3 \mathrm{~Hz}, \mathrm{H}_{3^{\prime}, 5^{\prime}}\right), 7.28(\mathrm{~d}, 1 \mathrm{H}, J$ $\left.=4.1 \mathrm{~Hz}, \mathrm{H}_{3}\right) ;{ }^{13} \mathrm{C}$ NMR $\left(125 \mathrm{MHz}, \mathrm{CDCl}_{3}\right) \delta=192.8(\mathrm{C}=\mathrm{O}), 145.2\left(\mathrm{C}_{3 \mathrm{a}}\right), 142.5\left(\mathrm{C}_{2}\right), 141.5\left(\mathrm{C}_{8 \mathrm{a}}\right)$, $141.3\left(\mathrm{C}_{1^{\prime}}\right), 139.5\left(\mathrm{C}_{6}\right), 139.1\left(\mathrm{C}_{8}\right), 138.5\left(\mathrm{C}_{4}\right), 131.1\left(\mathrm{C}_{4}\right), 129.5\left(\mathrm{C}_{2^{\prime}, 6^{\prime}}\right), 129.0\left(\mathrm{C}_{7}\right), 128.0\left(\mathrm{C}_{3^{\prime}, 5^{\prime}}\right), 127.4$ $\left(\mathrm{C}_{5}\right), 124.9\left(\mathrm{C}_{1}\right), 117.6\left(\mathrm{C}_{3}\right)$.

1-Benzoyl-3,6-di-tert-butylazulene (5b). The same procedure used for the preparation of $\mathbf{5 a}$ was adopted. The reaction of 1,6-di-tert-butylazulene (3b) (1.89 g, $7.86 \mathrm{mmol})$ and 4 (2.48 g, $16.6 \mathrm{mmol})$ with $\mathrm{POCl}_{3}(1.5 \mathrm{~mL}, 16 \mathrm{mmol})$ in refluxing THF $(40 \mathrm{~mL})$ for $4 \mathrm{~h}$ and column chromatography on silica gel with $\mathrm{CH}_{2} \mathrm{Cl}_{2}$ afforded $\mathbf{5 b}\left(2.24 \mathrm{~g}, 83 \%\right.$ ). purple crystals; mp $80.9-81.9^{\circ} \mathrm{C}$; $\mathrm{MS}(70 \mathrm{eV}) \mathrm{m} / z 344$ $\left(\mathrm{M}^{+}, 45 \%\right), 330$ (26), $329\left(\mathrm{M}^{+}-\mathrm{CH}_{3}, 100\right)$; IR (KBr disk) $v_{\max }$ 2965, $1618(\mathrm{C}=\mathrm{O}), 1576,1424,1376$, $1239 \mathrm{~cm}^{-1}$; UV-vis $\left(\mathrm{CH}_{2} \mathrm{Cl}_{2}\right) \lambda_{\max }, \mathrm{nm}(\log \varepsilon) 285$ (4.37), 326 (4.50), $401 \mathrm{sh}$ (4.07), 412 (4.08), 542 (2.77), $584 \operatorname{sh}(2.64), 644 \operatorname{sh}(2.07) ;{ }^{1} \mathrm{H}$ NMR $\left(400 \mathrm{MHz}, \mathrm{CDCl}_{3}\right) \delta=9.68\left(\mathrm{~d}, 1 \mathrm{H}, J=10.8 \mathrm{~Hz}, \mathrm{H}_{8}\right)$, $8.82\left(\mathrm{~d}, 1 \mathrm{H}, J=10.9 \mathrm{~Hz}, \mathrm{H}_{4}\right), 7.93\left(\mathrm{~s}, 1 \mathrm{H}, \mathrm{H}_{2}\right), 7.86\left(\mathrm{dd}, 1 \mathrm{H}, J=7.9,1.6 \mathrm{~Hz}, \mathrm{H}_{2,6}\right), 7.71(\mathrm{dd}, 1 \mathrm{H}, J=$ 10.8, $\left.1.9 \mathrm{~Hz}, \mathrm{H}_{7}\right), 7.64\left(\mathrm{dd}, 1 \mathrm{H}, J=10.9,1.9 \mathrm{~Hz}, \mathrm{H}_{5}\right), 7.45-7.45$ (m, 3H, $\left.\mathrm{H}_{3^{\prime}, 4^{\prime}, 5^{\prime}}\right), 1.55$ (s, 9H, 3-t-Bu), $1.45(\mathrm{~s}, 9 \mathrm{H}, 6-t-\mathrm{Bu}) ;{ }^{13} \mathrm{C}$ NMR $\left(100 \mathrm{MHz}, \mathrm{CDCl}_{3}\right) \delta=192.2(\mathrm{C}=\mathrm{O}), 163.5\left(\mathrm{C}_{6}\right), 141.9\left(\mathrm{C}_{8 \mathrm{a}}\right), 141.6$ $\left(\mathrm{C}_{1}\right), 139.6\left(\mathrm{C}_{2}\right), 139.1\left(\mathrm{C}_{3 \mathrm{a}}\right), 138.1\left(\mathrm{C}_{3}\right), 137.5\left(\mathrm{C}_{8}\right), 136.4\left(\mathrm{C}_{4}\right), 130.6\left(\mathrm{C}_{4}\right), 129.2\left(\mathrm{C}_{2^{\prime}, 6^{\prime}}\right), 127.8\left(\mathrm{C}_{3^{\prime}, 5^{\prime}}\right)$, 
$126.4\left(\mathrm{C}_{7}\right), 124.0\left(\mathrm{C}_{5}\right), 122.1\left(\mathrm{C}_{1}\right), 38.4(\mathrm{~s}, 6-t-\mathrm{Bu}), 32.7(\mathrm{~s}, 3-t-\mathrm{Bu}), 31.6(\mathrm{q}$ and q, 3- and 6-t-Bu). Anal. calcd for $\mathrm{C}_{25} \mathrm{H}_{28} \mathrm{O}$ : C, 87.16; H, 8.19. Found: C, 86.77; H, 8.25.

Ethyl (1-azulenyl)glyoxylate (11). The reaction of 3a (560 mg, $4.37 \mathrm{mmol})$ with 7 (210 $\mathrm{mg}, 0.973$ mmol) in the presence of sodium acetate $(210 \mathrm{mg}, 2.56 \mathrm{mmol})$ in $\mathrm{CH}_{2} \mathrm{Cl}_{2}(30 \mathrm{~mL})$ at $-78{ }^{\circ} \mathrm{C}$ for $4 \mathrm{~h}$ afforded 8a (18 mg, 7\%) and $\mathbf{1 1}(168 \mathrm{mg}, 76 \%)$ : red oil; MS (70 eV) m/z $228\left(\mathrm{M}^{+}, 13 \%\right), 155\left(\mathrm{M}^{+}-\right.$ $\left.\mathrm{CO}_{2} \mathrm{Et}, 100\right), 127\left(\mathrm{M}^{+}-\mathrm{COCO}_{2} \mathrm{Et}, 21\right)$; IR (neat) $v_{\max } 1694(\mathrm{C}=\mathrm{O}), 1426,1402,1228,1214,1176$, 1148, 1038, $772 \mathrm{~cm}^{-1}$; UV-vis $\left(\mathrm{CH}_{2} \mathrm{Cl}_{2}\right) \lambda_{\max }, \mathrm{nm}(\log \varepsilon) 231$ (4.27), $272 \mathrm{sh}$ (4.07), 280 (4.07), $304 \mathrm{sh}$ (4.37), 314 (4.47), $382 \mathrm{sh}$ (4.05), 397 (4.12), $484 \mathrm{sh}$ (2.79), 517 (2.87), $553 \mathrm{sh}$ (2.76), $600 \mathrm{sh}(2.28) ;{ }^{1} \mathrm{H}$ NMR $\left(600 \mathrm{MHz}, \mathrm{CDCl}_{3}\right) \delta=9.74\left(\mathrm{~d}, 1 \mathrm{H}, J=9.8 \mathrm{~Hz}, \mathrm{H}_{8}\right), 8.39\left(\mathrm{~d}, 1 \mathrm{H}, J=9.8 \mathrm{~Hz}, \mathrm{H}_{4}\right), 8.34(\mathrm{~d}, 1 \mathrm{H}, J=$ $\left.4.3 \mathrm{~Hz}, \mathrm{H}_{2}\right), 7.78\left(\mathrm{dd}, 1 \mathrm{H}, J=10.0,9.6 \mathrm{~Hz}, \mathrm{H}_{6}\right), 7.59\left(\mathrm{dd}, 1 \mathrm{H}, J=10.0,9.8 \mathrm{~Hz}, \mathrm{H}_{7}\right), 7.49$ (dd, $1 \mathrm{H}, J=$ 9.8, $\left.9.6 \mathrm{~Hz}, \mathrm{H}_{5}\right), 7.19\left(\mathrm{~d}, 1 \mathrm{H}, J=4.3 \mathrm{~Hz}, \mathrm{H}_{3}\right), 4.43\left(\mathrm{q}, 2 \mathrm{H}, J=7.1 \mathrm{~Hz}, 1-\mathrm{COCO}_{2} \mathrm{Et}\right), 1.41$ (t, $3 \mathrm{H}, J=7.1$

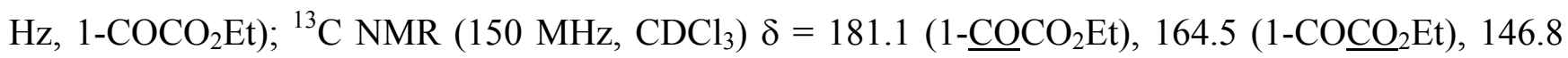
$\left(\mathrm{C}_{3 \mathrm{a}}\right), 142.4\left(\mathrm{C}_{2}\right.$ and $\left.\mathrm{C}_{8 \mathrm{a}}\right), 140.0\left(\mathrm{C}_{6}\right), 139.2\left(\mathrm{C}_{8}\right), 138.7\left(\mathrm{C}_{4}\right), 130.7\left(\mathrm{C}_{7}\right), 129.3\left(\mathrm{C}_{5}\right), 120.3\left(\mathrm{C}_{1}\right), 119.1$ $\left(\mathrm{C}_{3}\right), 61.7$ (t, 1-COCO $2 \mathrm{Et}$ ), 14.0 (q, 1- $\mathrm{COCO}_{2} \mathrm{Et}$ ). Anal. calcd for $\mathrm{C}_{14} \mathrm{H}_{12} \mathrm{O}_{3}: \mathrm{C}, 73.67 ; \mathrm{H}, 5.30$. Found: C, 73.90; H, 5.49.

$N, N$-Diethyl-2-(1-azulenyl)glyoxylamido (12). The reaction of 3a (548 mg, $4.28 \mathrm{mmol})$ with 7 (175 $\mathrm{mg}, 0.812 \mathrm{mmol})$ in the presence of triethylamine $(205 \mathrm{mg}, 2.03 \mathrm{mmol})$ in $\mathrm{CH}_{2} \mathrm{Cl}_{2}(30 \mathrm{~mL})$ at room temperature for 2 days afforded 8a (14 mg, 6\%), 9a (69 mg, 27\%), 11 (21 mg, 11\%), and 12 (71 mg, 34\%): dark brown crystals; mp $88.2-90.1{ }^{\circ} \mathrm{C}$; MS $(70 \mathrm{eV}) \mathrm{m} / \mathrm{z} 255\left(\mathrm{M}^{+}, 6 \%\right), 155\left(\mathrm{M}^{+}-\mathrm{CONEt}_{2}\right.$, 100); IR (KBr disk) $v_{\max } 1638(\mathrm{C}=\mathrm{O}), 1624(\mathrm{C}=\mathrm{O}), 1611(\mathrm{C}=\mathrm{O}), 1412,1397,1271 \mathrm{~cm}^{-1}$; UV-vis $\left(\mathrm{CH}_{2} \mathrm{Cl}_{2}\right) \lambda_{\max }, \mathrm{nm}$ (log $\left.\varepsilon\right) 235 \mathrm{sh}$ (4.23), 268 (3.99), $303 \mathrm{sh}$ (4.46), 314 (4.56), $382 \mathrm{sh}$ (4.08), 394 (4.10), $493 \mathrm{sh}(2.72), 522$ (2.80), $555 \mathrm{sh}(2.70), 612(2.18) ;{ }^{1} \mathrm{H}$ NMR $\left(400 \mathrm{MHz}, \mathrm{CDCl}_{3}\right) \delta=9.85(\mathrm{~d}, 1 \mathrm{H}, J=$ 
$\left.9.8 \mathrm{~Hz}, \mathrm{H}_{8}\right), 8.50\left(\mathrm{~d}, 1 \mathrm{H}, J=9.5 \mathrm{~Hz}, \mathrm{H}_{4}\right), 8.15$ (d, $\left.1 \mathrm{H}, J=4.3 \mathrm{~Hz}, \mathrm{H}_{2}\right), 7.89$ (dd, 1H, $J=10.0,9.8 \mathrm{~Hz}$, $\left.\mathrm{H}_{6}\right), 7.68\left(\mathrm{dd}, 1 \mathrm{H}, J=9.8,9.8 \mathrm{~Hz}, \mathrm{H}_{7}\right), 7.58\left(\mathrm{dd}, 1 \mathrm{H}, J=9.8,9.5 \mathrm{~Hz}, \mathrm{H}_{5}\right), 7.29\left(\mathrm{~d}, 1 \mathrm{H}, J=4.3 \mathrm{~Hz}, \mathrm{H}_{3}\right)$, 3.59 (q, 2H, $J=7.1 \mathrm{~Hz}, \mathrm{NEt}), 3.36$ (q, 2H, $J=7.1 \mathrm{~Hz}, \mathrm{NEt}), 1.32$ (t, 3H, $J=7.1 \mathrm{~Hz}, \mathrm{NEt}), 1.17$ (t, 3H, $J=7.1 \mathrm{~Hz}, \mathrm{NEt}) ;{ }^{13} \mathrm{C} \mathrm{NMR}\left(100 \mathrm{MHz}, \mathrm{CDCl}_{3}\right) \delta=188.3\left(1\right.$ - $\left.\underline{\mathrm{COCONEt}_{2}}\right), 168.0\left(1-\mathrm{COCONEt}_{2}\right), 146.6$ $\left(\mathrm{C}_{3 \mathrm{a}}\right), 142.2\left(\mathrm{C}_{2}\right), 141.6\left(\mathrm{C}_{8 \mathrm{a}}\right), 140.0\left(\mathrm{C}_{6}\right), 139.3\left(\mathrm{C}_{8}\right), 138.9\left(\mathrm{C}_{4}\right), 130.3\left(\mathrm{C}_{7}\right), 128.8\left(\mathrm{C}_{5}\right), 121.3\left(\mathrm{C}_{1}\right)$, $119.0\left(\mathrm{C}_{3}\right), 42.2$ (t, NEt), 38.6 (t, NEt), 14.2 (q, NEt), 12.9 (q, NEt). Anal. calcd for $\mathrm{C}_{16} \mathrm{H}_{17} \mathrm{NO}_{2}: \mathrm{C}_{\text {, }}$ 75.27; H, 6.71; N, 5.49. Found: C, 75.37; H, 6.79; N, 5.41.

Bis(3,6-di-tert-butyl-1-azulenyl) ketone (8b). The general procedure was followed by using 1,6-di-tert-butylazulene (3b) (1.39 g, $5.78 \mathrm{mmol})$ and 7 (552 mg, $2.56 \mathrm{mmol})$ in $\mathrm{CH}_{2} \mathrm{Cl}_{2}(40 \mathrm{~mL})$ at room temperature for $5.5 \mathrm{~h}$. Chromatographic purification on silica gel with ethyl acetate $/ \mathrm{CH}_{2} \mathrm{Cl}_{2}$ afforded $\mathbf{8 b}$ (926 mg, 71\%) and bis(3,6-di-tert-butyl-1-azulenyl) diketone (9b) (59 mg, 4\%). Recrystallization of $\mathbf{8 b}$ from hexane afforded fine incursion crystals with hexane in the ratio of 2:1 (8b:hexane; determined by NMR measurements), which did not decompose in vacuum desiccator.

8b: ${ }^{13}$ purple crystals; mp $146.0-147.5^{\circ} \mathrm{C}$ (hexane inclusion crystals, which melt with evolution of a gas). [lit. ${ }^{13} \mathrm{mp} 212.8-214.2^{\circ} \mathrm{C}$ (non-inclusion crystals)].

9b: reddish purple crystals; mp $272.2-274.9^{\circ} \mathrm{C}$; $\mathrm{MS}(70 \mathrm{eV}) \mathrm{m} / z 534\left(\mathrm{M}^{+}, 4.3 \%\right), 268(20), 267\left(\mathrm{M}^{+}\right.$ $\left.-\mathrm{COC}_{18} \mathrm{H}_{23}, 100\right)$; IR (KBr disk) $v_{\max } 2965,1605(\mathrm{C}=\mathrm{O}), 1576,1426,1397,1379,1364,1221,648$ $\mathrm{cm}^{-1}$; UV-vis $\left(\mathrm{CH}_{2} \mathrm{Cl}_{2}\right) \lambda_{\max }, \mathrm{nm}(\log \varepsilon) 277$ (4.34), $315 \mathrm{sh}$ (4.62), 329 (4.67), $405 \mathrm{sh}$ (4.33), 423 (4.46), $517 \mathrm{sh}(3.22), 575 \mathrm{sh}(3.03), 625$ (2.44); ${ }^{1} \mathrm{H} \mathrm{NMR}\left(400 \mathrm{MHz}, \mathrm{CDCl}_{3}\right) \delta=9.94\left(\mathrm{~d}, 2 \mathrm{H}, J=10.6 \mathrm{~Hz}, \mathrm{H}_{8}\right)$, $8.81\left(\mathrm{~d}, 2 \mathrm{H}, J=10.9 \mathrm{~Hz}, \mathrm{H}_{4}\right), 8.09\left(\mathrm{~s}, 2 \mathrm{H}, \mathrm{H}_{2}\right), 7.83\left(\mathrm{dd}, 2 \mathrm{H}, J=10.6,1.7 \mathrm{~Hz}, \mathrm{H}_{7}\right), 7.71(\mathrm{dd}, 2 \mathrm{H}, J=$ 10.9, $\left.1.7 \mathrm{~Hz}, \mathrm{H}_{5}\right), 1.52(\mathrm{~s}, 18 \mathrm{H}, 3-t-\mathrm{Bu}), 1.51(\mathrm{~s}, 18 \mathrm{H}, 6-t-\mathrm{Bu}) ;{ }^{13} \mathrm{C} \mathrm{NMR}\left(100 \mathrm{MHz}, \mathrm{CDCl}_{3}\right) \delta=191.4$ $(\mathrm{C}=\mathrm{O}), 163.9\left(\mathrm{C}_{6}\right), 142.8\left(\mathrm{C}_{8 \mathrm{a}}\right), 140.9\left(\mathrm{C}_{3 \mathrm{a}}\right), 140.0\left(\mathrm{C}_{2}\right), 139.6\left(\mathrm{C}_{3}\right), 138.1\left(\mathrm{C}_{8}\right), 136.6\left(\mathrm{C}_{4}\right), 127.8\left(\mathrm{C}_{7}\right)$, $125.3\left(\mathrm{C}_{5}\right), 119.2\left(\mathrm{C}_{1}\right), 38.7(\mathrm{~s}, 6-t-\mathrm{Bu}), 32.9(\mathrm{~s}, 3-t-\mathrm{Bu}), 31.8(\mathrm{q}, 6-t-\mathrm{Bu}), 31.6(\mathrm{q}, 3-t-\mathrm{Bu})$. Anal. calcd 
for $\mathrm{C}_{38} \mathrm{H}_{46} \mathrm{O}_{2}$ : C, 85.35; H, 8.67. Found: C, 85.18; H, 8.89.

Bis(3-methyl-1-azulenyl) ketone (8c). The general procedure was followed by using 1-methylazulene (3c) $(741 \mathrm{mg}, 5.21 \mathrm{mmol})$ and $7(504 \mathrm{mg}, 2.34 \mathrm{mmol})$ in $\mathrm{CH}_{2} \mathrm{Cl}_{2}(50 \mathrm{~mL})$ at room temperature for $1 \mathrm{~h}$. Chromatographic purification on silica gel with ethyl acetate $/ \mathrm{CH}_{2} \mathrm{Cl}_{2}$ and $\mathrm{GPC}$ with $\mathrm{CHCl}_{3}$ afforded 8c (440 mg, 61\%) and bis(3-methyl-1-azulenyl) diketone (9c) (22 mg, 3\%).

8c: ${ }^{10 b}$ green needles; mp $179.2-179.8^{\circ} \mathrm{C}$. [lit. ${ }^{10 \mathrm{~b}} \mathrm{mp} 163{ }^{\circ} \mathrm{C}$ ]; MS $(70 \mathrm{eV}) \mathrm{m} / z 310\left(\mathrm{M}^{+}, 100 \%\right), 309$ (24), $295\left(\mathrm{M}^{+}-\mathrm{CH}_{3}, 54\right), 267$ (22), 141 (23); IR (KBr disk) v $v_{\max }$ 1588, 1431, 1190, 781, $729 \mathrm{~cm}^{-1}$; UV-vis $\left(\mathrm{CH}_{2} \mathrm{Cl}_{2}\right) \lambda_{\max }, \mathrm{nm}(\log \varepsilon) 234$ (4.57), 270 sh (4.42), 291 (4.64), 313 sh (4.50), 321 (4.50), 424 (4.41), $531 \mathrm{sh}(2.96), 569$ (3.02), $621 \mathrm{sh}(2.86), 681 \mathrm{sh}(2.32) ;{ }^{1} \mathrm{H}$ NMR $\left(400 \mathrm{MHz}, \mathrm{CDCl}_{3}\right) \delta=9.53(\mathrm{~d}$, $\left.2 \mathrm{H}, J=9.8 \mathrm{~Hz}, \mathrm{H}_{8}\right), 8.36\left(\mathrm{~d}, 2 \mathrm{H}, J=9.8 \mathrm{~Hz}, \mathrm{H}_{4}\right), 8.02\left(\mathrm{~s}, 2 \mathrm{H}, \mathrm{H}_{2}\right), 7.71\left(\mathrm{dd}, 2 \mathrm{H}, J=9.8,9.8 \mathrm{~Hz}, \mathrm{H}_{6}\right)$, $7.42\left(\mathrm{dd}, 2 \mathrm{H}, J=9.8,9.8 \mathrm{~Hz}, \mathrm{H}_{7}\right), 7.35\left(\mathrm{dd}, 2 \mathrm{H}, J=9.8,9.8 \mathrm{~Hz}, \mathrm{H}_{5}\right), 2.65(\mathrm{~s}, 6 \mathrm{H}, 3-\mathrm{Me}) ;{ }^{13} \mathrm{C} \mathrm{NMR}$ $\left(100 \mathrm{MHz}, \mathrm{CDCl}_{3}\right) \delta=188.8(\mathrm{C}=\mathrm{O}), 142.3\left(\mathrm{C}_{2}\right), 140.9\left(\mathrm{C}_{3 \mathrm{a}}\right.$ and $\left.\mathrm{C}_{8 \mathrm{a}}\right), 138.9\left(\mathrm{C}_{6}\right), 137.9\left(\mathrm{C}_{8}\right), 135.1\left(\mathrm{C}_{4}\right)$, $127.1\left(\mathrm{C}_{7}\right.$ and $\left.\mathrm{C}_{1}\right), 125.1\left(\mathrm{C}_{3}\right), 125.0\left(\mathrm{C}_{5}\right), 12.7$ (3-Me). Anal. calcd for $\mathrm{C}_{23} \mathrm{H}_{18} \mathrm{O}$ : $\mathrm{C}, 89.00 ; \mathrm{H}, 5.85$. Found: C, 89.15; H, 5.99.

9c: purple needles; mp $267.7-268.4{ }^{\circ} \mathrm{C}$; $\mathrm{MS}(70 \mathrm{eV}) \mathrm{m} / z 338\left(\mathrm{M}^{+}, 10 \%\right), 169\left(\mathrm{M}^{+}-\mathrm{COC}_{11} \mathrm{H}_{9}, 100\right)$; IR (KBr disk) $v_{\max } 1613(\mathrm{C}=\mathrm{O}), 1418,1389,1375 \mathrm{~cm}^{-1}$; UV-vis $\left(\mathrm{CH}_{2} \mathrm{Cl}_{2}\right) \lambda_{\max }, \mathrm{nm}(\log \varepsilon) 233(4.58)$, 274 (4.36), $306 \mathrm{sh}$ (4.60), 322 (4.68), $400 \mathrm{sh}$ (4.32), 423 (4.48), $518 \mathrm{sh}(3.12), 548$ (3.13), 598 sh (2.94), $654 \mathrm{sh}(2.36) ;{ }^{1} \mathrm{H}$ NMR $\left(500 \mathrm{MHz}, \mathrm{CDCl}_{3}\right) \delta=9.95\left(\mathrm{~d}, 2 \mathrm{H}, J=9.7 \mathrm{~Hz}, \mathrm{H}_{8}\right), 8.40(\mathrm{~d}, 2 \mathrm{H}, J=9.8 \mathrm{~Hz}$, $\left.\mathrm{H}_{4}\right), 8.10\left(\mathrm{~s}, 2 \mathrm{H}, \mathrm{H}_{2}\right), 7.85\left(\mathrm{dd}, 2 \mathrm{H}, J=9.8,9.8 \mathrm{~Hz}, \mathrm{H}_{6}\right), 7.66\left(\mathrm{dd}, 2 \mathrm{H}, J=9.8,9.7 \mathrm{~Hz}, \mathrm{H}_{7}\right), 7.53(\mathrm{dd}, 2 \mathrm{H}$, $\left.J=9.8,9.8 \mathrm{~Hz}, \mathrm{H}_{5}\right), 2.54(\mathrm{~s}, 6 \mathrm{H}, 3-\mathrm{Me}) ;{ }^{13} \mathrm{C} \mathrm{NMR}\left(125 \mathrm{MHz}, \mathrm{CDCl}_{3}\right) \delta=191.5(\mathrm{C}=\mathrm{O}), 143.6\left(\mathrm{C}_{3 \mathrm{a}}\right)$, $143.4\left(\mathrm{C}_{2}\right), 142.5\left(\mathrm{C}_{8 \mathrm{a}}\right), 139.5\left(\mathrm{C}_{6}\right), 139.0\left(\mathrm{C}_{8}\right), 135.6\left(\mathrm{C}_{4}\right), 129.8\left(\mathrm{C}_{7}\right), 127.5\left(\mathrm{C}_{5}\right), 127.0\left(\mathrm{C}_{3}\right), 119.7$ $\left(\mathrm{C}_{1}\right), 12.5$ (3-Me). Anal. calcd for $\mathrm{C}_{24} \mathrm{H}_{18} \mathrm{O}_{2}$ : C, 85.18; H, 5.36. Found: C, 85.06; H, 5.49. 
Bis(6-tert-butyl-1-azulenyl) ketone (8d). The general procedure was followed by using 6-tert-butylazulene (3d) $(1.14 \mathrm{~g}, 6.19 \mathrm{mmol})$ and $7(664 \mathrm{mg}, 3.08 \mathrm{mmol})$ in $\mathrm{CH}_{2} \mathrm{Cl}_{2}(60 \mathrm{~mL})$ at room temperature for $1 \mathrm{~h}$. Chromatographic purification on silica gel with ethyl acetate $/ \mathrm{CH}_{2} \mathrm{Cl}_{2}$ and $\mathrm{GPC}$ with $\mathrm{CHCl}_{3}$ afforded $\mathbf{8 d}(208 \mathrm{mg}, 17 \%)$ and bis(6-tert-butyl-1-azulenyl) diketone (9d) (15 mg, 1\%).

8d: reddish brown crystals; $\mathrm{mp} 162.7-163.6^{\circ} \mathrm{C}$; $\mathrm{MS}(70 \mathrm{eV}) \mathrm{m} / z 394\left(\mathrm{M}^{+}, 98 \%\right), 338(31), 337\left(\mathrm{M}^{+}-\right.$ $t$-Bu, 100); IR (KBr disk) $v_{\max } 2963,1584,1489,1443,1401,1291,1242,1208,845,814,756 \mathrm{~cm}^{-1}$; UV-vis $\left(\mathrm{CH}_{2} \mathrm{Cl}_{2}\right) \lambda_{\max }, \mathrm{nm}(\log \varepsilon) 233$ sh (log $\varepsilon$ 4.52), 289 (4.66), 326 (4.58), $346 \mathrm{sh}$ (4.31), $394 \mathrm{sh}$ (4.39), 4.09 (4.44), 495 sh (3.08), 527 (3.14), 573 sh (2.98), 627 sh (2.37); ${ }^{1} \mathrm{H}$ NMR (500 MHz, $\left.\mathrm{CDCl}_{3}\right)$ $\delta=9.58\left(\mathrm{~d}, 2 \mathrm{H}, J=10.7 \mathrm{~Hz}, \mathrm{H}_{8}\right), 8.43\left(\mathrm{~d}, 2 \mathrm{H}, J=10.5 \mathrm{~Hz}, \mathrm{H}_{4}\right), 8.14\left(\mathrm{~d}, 2 \mathrm{H}, J=4.0 \mathrm{~Hz}, \mathrm{H}_{2}\right), 7.72(\mathrm{dd}$, $\left.2 \mathrm{H}, J=10.7,1.9 \mathrm{~Hz}, \mathrm{H}_{7}\right), 7.61\left(\mathrm{dd}, 2 \mathrm{H}, J=10.5,1.9 \mathrm{~Hz}, \mathrm{H}_{5}\right), 7.25\left(\mathrm{~d}, 2 \mathrm{H}, J=4.0 \mathrm{~Hz}, \mathrm{H}_{3}\right), 1.49(\mathrm{~s}, 18 \mathrm{H}$, 6-t-Bu); ${ }^{13} \mathrm{C}$ NMR $\left(125 \mathrm{MHz}, \mathrm{CDCl}_{3}\right) \delta=189.1(\mathrm{C}=\mathrm{O}), 163.4\left(\mathrm{C}_{6}\right), 143.1\left(\mathrm{C}_{3 \mathrm{a}}\right), 140.9\left(\mathrm{C}_{2}\right), 139.5\left(\mathrm{C}_{8 \mathrm{a}}\right)$, 137.6 $\left(\mathrm{C}_{8}\right), 137.2\left(\mathrm{C}_{4}\right), 128.5\left(\mathrm{C}_{1}\right), 125.8\left(\mathrm{C}_{7}\right), 124.7\left(\mathrm{C}_{5}\right), 116.6\left(\mathrm{C}_{3}\right), 38.8(\mathrm{~s}, 6-t-\mathrm{Bu}), 31.9(\mathrm{q}, 6-t-\mathrm{Bu})$. Anal. calcd for $\mathrm{C}_{29} \mathrm{H}_{30} \mathrm{O}$ : C, 88.28; H, 7.66. Found: C, 88.31; H, 7.79.

9d: red crystals; mp $253.0-253.9{ }^{\circ} \mathrm{C}$; $\mathrm{MS}(70 \mathrm{eV}) \mathrm{m} / z 422\left(\mathrm{M}^{+}, 7 \%\right), 211\left(\mathrm{M}^{+}-\mathrm{COC}_{14} \mathrm{H}_{15}, 100\right)$; IR $(\mathrm{KBr}$ disk $) v_{\max } 1620(\mathrm{C}=\mathrm{O}), 1601,1578,1491,1441,1399,1291,1242,855,646 \mathrm{~cm}^{-1}$; UV-vis $\left(\mathrm{CH}_{2} \mathrm{Cl}_{2}\right) \lambda_{\max }$, nm (log $\left.\varepsilon\right) 238 \mathrm{sh}$ (4.51), $262 \mathrm{sh}$ (4.22), 271 (4.29), $305 \mathrm{sh}$ (4.63), 325 (4.75), $384 \mathrm{sh}$ (4.39), 405 (4.56), 507 (3.34), $550 \mathrm{sh}$ (3.16), $601 \mathrm{sh}(2.52) ;{ }^{1} \mathrm{H}$ NMR $\left(500 \mathrm{MHz}, \mathrm{CDCl}_{3}\right) \delta=9.93(\mathrm{~d}$, $\left.2 \mathrm{H}, J=10.6 \mathrm{~Hz}, \mathrm{H}_{8}\right), 8.44\left(\mathrm{~d}, 2 \mathrm{H}, J=10.6 \mathrm{~Hz}, \mathrm{H}_{4}\right), 8.17\left(\mathrm{~d}, 2 \mathrm{H}, J=4.3 \mathrm{~Hz}, \mathrm{H}_{2}\right), 7.91(\mathrm{dd}, 2 \mathrm{H}, J=10.7$, $\left.1.9 \mathrm{~Hz}, \mathrm{H}_{7}\right), 7.75\left(\mathrm{dd}, 2 \mathrm{H}, J=10.6,1.9 \mathrm{~Hz}, \mathrm{H}_{5}\right), 7.16\left(\mathrm{~d}, 2 \mathrm{H}, J=4.3 \mathrm{~Hz}, \mathrm{H}_{3}\right), 1.51(\mathrm{~s}, 18 \mathrm{H}, 6-t-\mathrm{Bu}) ;{ }^{13} \mathrm{C}$ $\operatorname{NMR}\left(125 \mathrm{MHz}, \mathrm{CDCl}_{3}\right) \delta=191.7(\mathrm{C}=\mathrm{O}), 164.4\left(\mathrm{C}_{6}\right), 145.3\left(\mathrm{C}_{3 \mathrm{a}}\right), 142.0\left(\mathrm{C}_{2}\right), 140.8\left(\mathrm{C}_{8 \mathrm{a}}\right), 138.6\left(\mathrm{C}_{8}\right)$, 137.6 $\left(\mathrm{C}_{4}\right), 128.2\left(\mathrm{C}_{7}\right), 127.1\left(\mathrm{C}_{5}\right), 121.4\left(\mathrm{C}_{1}\right), 118.4\left(\mathrm{C}_{3}\right), 39.0(\mathrm{~s}$, 6-t-Bu), $31.9(\mathrm{q}, 6-t$-Bu). Anal. calcd for $\mathrm{C}_{30} \mathrm{H}_{30} \mathrm{O}_{2}$ : C, 85.27; H, 7.16. Found: C, 85.20; H, 7.24. 
2-Azulenyl-2-phenyl-4,5-dimethyldihydrothiopyran (16). A solution of 1-thiobenzoylazulene (1a) (226 mg, $0.910 \mathrm{mmol}$ ) and 2,3-dimethyl-1,3-butadiene (15) (439 mg, $5.34 \mathrm{mmol})$ in $\mathrm{CHCl}_{3}(10 \mathrm{~mL})$ was refluxed for $72 \mathrm{~h}$. After the solvent was removed under reduced pressure, the residue was purified by column chromatography on silica gel with $\mathrm{CH}_{2} \mathrm{Cl}_{2}$ and $\mathrm{GPC}$ with $\mathrm{CHCl}_{3}$ to afford 16 (156 $\left.\mathrm{mg}, 52 \%\right)$. blue crystals; mp $34.5-38.2{ }^{\circ} \mathrm{C}$; $\mathrm{MS}(70 \mathrm{eV}) \mathrm{m} / \mathrm{z} 330\left(\mathrm{M}^{+}, 28 \%\right), 297$ (31), 249 (22), $248\left(\mathrm{M}^{+}-\mathrm{C}_{6} \mathrm{H}_{10}\right.$, 100), 247 (72); IR (KBr disk) $v_{\max } 1399,766,743,700 \mathrm{~cm}^{-1}$; UV-vis $\left(\mathrm{CH}_{2} \mathrm{Cl}_{2}\right) \lambda_{\max }, \mathrm{nm}(\log \varepsilon) 234$ (4.26), 292 (4.49), 343 sh (3.59), 352 (3.62), 370 (3.49), 597 (2.49), 642 sh (2.41), 704 sh (1.97); ${ }^{1} \mathrm{H}$ $\operatorname{NMR}\left(500 \mathrm{MHz}, \mathrm{CDCl}_{3}\right) \delta=8.31\left(\mathrm{~d}, 1 \mathrm{H}, J=9.8 \mathrm{~Hz}, \mathrm{H}_{8^{\prime}}\right), 8.26\left(\mathrm{~d}, 1 \mathrm{H}, J=9.5 \mathrm{~Hz}, \mathrm{H}_{4^{\prime}}\right), 7.74(\mathrm{~d}, 1 \mathrm{H}, J$ $\left.=4.0 \mathrm{~Hz}, \mathrm{H}_{2^{\prime}}\right), 7.47\left(\mathrm{dd}, 1 \mathrm{H}, J=10.0,9.8 \mathrm{~Hz}, \mathrm{H}_{6^{\prime}}\right), 7.32\left(\mathrm{~m}, 2 \mathrm{H}, \mathrm{H}_{2^{\prime \prime}}, 6^{\prime \prime}\right), 7.23\left(\mathrm{~d}, 1 \mathrm{H}, J=4.0 \mathrm{~Hz}, \mathrm{H}_{3^{\prime}}\right)$, $7.23\left(\mathrm{~m}, 2 \mathrm{H}, \mathrm{H}_{3^{\prime \prime}, 5^{\prime \prime}}\right), 7.17\left(\mathrm{tt}, 1 \mathrm{H}, J=7.2,1.3 \mathrm{~Hz}, \mathrm{H}_{4}\right), 7.08$ (dd, $\left.1 \mathrm{H}, J=9.8,9.5 \mathrm{~Hz}, \mathrm{H}_{5^{\prime}}\right), 6.89$ (dd, $1 \mathrm{H}$, $\left.J=10.0,9.8 \mathrm{~Hz}, \mathrm{H}_{7^{\prime}}\right), 3.04\left(\mathrm{~d}, 1 \mathrm{H}, J=17.7 \mathrm{~Hz}, \mathrm{H}_{5}\right), 2.95\left(\mathrm{~d}, 1 \mathrm{H}, J=17.7 \mathrm{~Hz}, \mathrm{H}_{5}\right), 2.73(\mathrm{~d}, 1 \mathrm{H}, J=16.9$ $\left.\mathrm{Hz}, \mathrm{H}_{2}\right), 2.47\left(\mathrm{~d}, 1 \mathrm{H}, J=16.9 \mathrm{~Hz}, \mathrm{H}_{2}\right), 1.81(\mathrm{~s}, 3 \mathrm{H}, 4-\mathrm{Me}), 1.65$ (s, 3H, 3-Me); ${ }^{13} \mathrm{C} \mathrm{NMR}(125 \mathrm{MHz}$, $\left.\mathrm{CDCl}_{3}\right) \delta=147.8\left(\mathrm{C}_{1^{\prime \prime}}\right), 141.7\left(\mathrm{C}_{8^{\prime} \mathrm{a}}\right), 138.2\left(\mathrm{C}_{2^{\prime}}\right), 137.4\left(\mathrm{C}_{6^{\prime}}\right), 137.1\left(\mathrm{C}_{8^{\prime}}\right), 136.6\left(\mathrm{C}_{4^{\prime}}\right), 134.6\left(\mathrm{C}_{3^{\prime} \mathrm{a}}\right), 131.6$ $\left(\mathrm{C}_{1^{\prime}}\right), 128.1\left(\mathrm{C}_{3^{\prime \prime}, 5^{\prime \prime}}\right), 127.0\left(\mathrm{C}_{2^{\prime \prime}, 6^{\prime \prime}}\right), 126.9\left(\mathrm{C}_{3}\right.$ or $\left.\mathrm{C}_{4}\right), 126.4\left(\mathrm{C}_{4 "}\right), 123.4\left(\mathrm{C}_{3}\right.$ or $\left.\mathrm{C}_{4}\right), 122.9\left(\mathrm{C}_{5^{\prime}}\right), 121.8$ $\left(\mathrm{C}_{7^{\prime}}\right), 115.1\left(\mathrm{C}_{3^{\prime}}\right), 51.1\left(\mathrm{C}_{6}\right), 49.3\left(\mathrm{C}_{5}\right), 31.6\left(\mathrm{C}_{2}\right), 19.9$ (4-Me), 19.2 (3-Me). HRMS calcd for $\mathrm{C}_{23} \mathrm{H}_{22} \mathrm{~S}$ 330.1442, found 330.1447. Anal. calcd for $\mathrm{C}_{23} \mathrm{H}_{22} \mathrm{~S}$ : C, 83.59; H, 6.71; S, 9.70. Found: C, 83.30; H, $6.91 ; \mathrm{S}, 9.65$.

3,6-Di-tert-butyl-1-thiobenzoylazulene (1b). The general procedure was followed by using 1-benzoyl-3,6-di-tert-butylazulene (5b) (207 mg, $0.601 \mathrm{mmol}), \mathrm{P}_{2} \mathrm{~S}_{5}(661 \mathrm{mg}, 2.97 \mathrm{mmol})$, and triethylamine $(1 \mathrm{~mL})$ in $\mathrm{CHCl}_{3}(25 \mathrm{~mL})$ at the room temperature for $3 \mathrm{~h}$. Chromatographic purification on silica gel with $60 \% \mathrm{CH}_{2} \mathrm{Cl}_{2} /$ hexane afforded $\mathbf{1 b}(141 \mathrm{mg}, 65 \%)$. brown crystals; $\mathrm{mp} 62.0-65.0{ }^{\circ} \mathrm{C}$; MS (70 eV) m/z $360\left(\mathrm{M}^{+}, 28 \%\right), 359(29), 345\left(\mathrm{M}^{+}-\mathrm{CH}_{3}, 29\right), 344$ (49), 330 (26), $329\left(\mathrm{M}^{+}-\mathrm{S}+\mathrm{H}\right.$, 100), $303\left(\mathrm{M}^{+}-t\right.$-Bu, 49), 105 (22); IR (KBr disk) $v_{\max } 2963,1578,1445,1422,1370,1238,1219$ 
$\mathrm{cm}^{-1}$; UV-vis $\left(\mathrm{CH}_{2} \mathrm{Cl}_{2}\right) \lambda_{\max }, \mathrm{nm}(\log \varepsilon) 239$ (4.42), 297 (4.39), 319 sh (4.21), 358 sh (4.01), 479 (4.12), $590 \mathrm{sh}(3.11) ;{ }^{1} \mathrm{H}$ NMR $\left(600 \mathrm{MHz}, \mathrm{CDCl}_{3}\right) \delta=9.50\left(\mathrm{~d}, 1 \mathrm{H}, J=10.9 \mathrm{~Hz}, \mathrm{H}_{8}\right), 8.78(\mathrm{~d}, 1 \mathrm{H}, J=11.0 \mathrm{~Hz}$, $\left.\mathrm{H}_{4}\right), 7.86\left(\mathrm{~s}, 1 \mathrm{H}, \mathrm{H}_{2}\right), 7.72\left(\mathrm{dd}, 1 \mathrm{H}, J=10.9,1.9 \mathrm{~Hz}, \mathrm{H}_{7}\right), 7.72\left(\mathrm{dd}, 1 \mathrm{H}, J=11.0,1.9 \mathrm{~Hz}, \mathrm{H}_{5}\right), 7.64(\mathrm{dd}$, $\left.2 \mathrm{H}, J=8.1,1.2 \mathrm{~Hz}, \mathrm{H}_{2^{\prime}, 6^{\prime}}\right), 7.48\left(\mathrm{tt}, 1 \mathrm{H}, J=7.4,1.2 \mathrm{~Hz}, \mathrm{H}_{4^{\prime}}\right), 7.39\left(\mathrm{dd}, 2 \mathrm{H}, J=8.1,7.4 \mathrm{~Hz}, \mathrm{H}_{3^{\prime}, 5^{\prime}}\right), 1.54$ (s, 9H, 3-t-Bu), $1.48(\mathrm{~s}, 9 \mathrm{H}, 6-t-\mathrm{Bu}) ;{ }^{13} \mathrm{C} \mathrm{NMR}\left(150 \mathrm{MHz}, \mathrm{CDCl}_{3}\right) \delta=222.9(\mathrm{C}=\mathrm{S}), 164.8\left(\mathrm{C}_{6}\right), 150.8$ $\left(\mathrm{C}_{1^{\prime}}\right), 143.1\left(\mathrm{C}_{3 \mathrm{a}}\right), 142.5\left(\mathrm{C}_{8 \mathrm{a}}\right), 139.5\left(\mathrm{C}_{2}\right.$ and $\left.\mathrm{C}_{3}\right), 138.5\left(\mathrm{C}_{8}\right), 136.9\left(\mathrm{C}_{4}\right), 135.9\left(\mathrm{C}_{1}\right), 130.0\left(\mathrm{C}_{4^{\prime}}\right), 129.3$ $\left(\mathrm{C}_{2^{\prime}, 6^{\prime}}\right), 128.9\left(\mathrm{C}_{7}\right), 127.7\left(\mathrm{C}_{3^{\prime}, 5^{\prime}}\right), 125.7\left(\mathrm{C}_{5}\right), 38.7(\mathrm{~s}, 6-t-\mathrm{Bu}), 32.8(\mathrm{~s}, 3-t-\mathrm{Bu}), 31.7(\mathrm{q}, 6-t-\mathrm{Bu}), 31.5(\mathrm{q}$, 3-t-Bu). Anal. calcd for $\mathrm{C}_{25} \mathrm{H}_{28} \mathrm{~S}: \mathrm{C}, 83.28 ; \mathrm{H}, 7.83$; S, 8.89. Found: C, 82.99; H, 7.85; S, 9.10.

2-Phenyl-4,7-di-tert-butyl-3H-azuleno[8,1-b,c]thiophene (13b). The reaction of $\mathbf{5 b}(290 \mathrm{mg}, 0.842$ $\mathrm{mmol})$ with Lawesson's reagent $(283 \mathrm{mg}, 0.700 \mathrm{mmol})$ in toluene $(20 \mathrm{~mL})$ at room temperature for $3 \mathrm{~h}$, following chromatographic purification on $\mathrm{Al}_{2} \mathrm{O}_{3}$ with $40 \% \mathrm{CH}_{2} \mathrm{Cl}_{2} /$ hexane, afforded $\mathbf{1 3 b}$ (186 mg, 89\%). red crystals; mp $114.4-117.5{ }^{\circ} \mathrm{C}$; MS (70 eV) m/z $360\left(\mathrm{M}^{+}, 34 \%\right), 359$ (22), $345\left(\mathrm{M}^{+}-\mathrm{CH}_{3}\right.$, 27), 304 (26), $303\left(\mathrm{M}^{+}-t\right.$-Bu, 100); IR (KBr disk) v $v_{\max } 2968,2908,1488,1244,1226,754,688 \mathrm{~cm}^{-1}$; UV-vis $\left(\mathrm{CH}_{2} \mathrm{Cl}_{2}\right) \lambda_{\max }, \mathrm{nm}(\log \varepsilon) 245$ (4.22), $313 \mathrm{sh}$ (4.48), 323 (4.50), 340 sh (4.19), 425 sh (3.16), $459 \mathrm{sh}$ (3.33), 490 (3.41), 524 (3.36), $568 \mathrm{sh}$ (3.13), $620 \mathrm{sh}(2.59) ;{ }^{1} \mathrm{H}$ NMR $\left(500 \mathrm{MHz}, \mathrm{CDCl}_{3}\right) \delta=$ $7.49\left(\mathrm{~d}, 2 \mathrm{H}, J=7.6 \mathrm{~Hz}, \mathrm{H}_{2^{\prime}, 6^{\prime}}\right), 7.37\left(\mathrm{dd}, 2 \mathrm{H}, J=7.9,7.6 \mathrm{~Hz}, \mathrm{H}_{3^{\prime}, 5^{\prime}}\right), 7.24\left(\mathrm{t}, 1 \mathrm{H}, J=7.9 \mathrm{~Hz}, \mathrm{H}_{4}{ }^{\prime}\right), 6.45$ $\left(\mathrm{d}, 1 \mathrm{H}, J=12.8 \mathrm{~Hz}, \mathrm{H}_{5}\right), 5.97\left(\mathrm{~s}, 1 \mathrm{H}, \mathrm{H}_{8}\right), 5.66\left(\mathrm{~d}, 1 \mathrm{H}, J=12.8 \mathrm{~Hz}, \mathrm{H}_{6}\right), 3.45\left(\mathrm{~s}, 2 \mathrm{H}, \mathrm{H}_{3}\right), 1.25(\mathrm{~s}, 9 \mathrm{H}$, 4-t-Bu), $1.11(\mathrm{~s}, 9 \mathrm{H}, 7-t-\mathrm{Bu}) ;{ }^{13} \mathrm{C} \mathrm{NMR}\left(125 \mathrm{MHz}, \mathrm{CDCl}_{3}\right) \delta=155.2\left(\mathrm{C}_{8 \mathrm{~b}}\right), 144.7\left(\mathrm{C}_{4}\right), 144.6\left(\mathrm{C}_{7}\right)$,

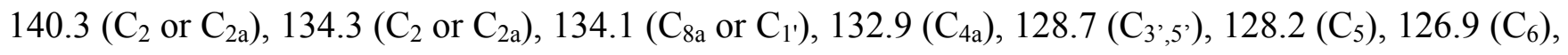

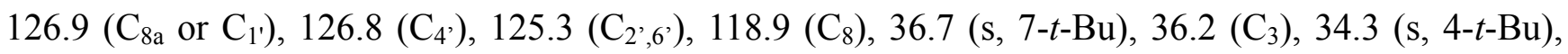
30.2 (q, 4-t-Bu), 29.4 (q, 7-t-Bu). Anal. calcd for $\mathrm{C}_{25} \mathrm{H}_{28} \mathrm{~S} \cdot 1 / 2 \mathrm{H}_{2} \mathrm{O}: \mathrm{C}, 81.25 ; \mathrm{H}, 7.91 ; \mathrm{S}, 8.68$. Found: C, 81.20; H, 7.84; S, 9.13. 
Di(1-azulenyl) thioketone (2a). The general procedure was followed by using di(1-azulenyl) ketone (8a) $(320 \mathrm{mg}, 1.13 \mathrm{mmol}), \mathrm{P}_{2} \mathrm{~S}_{5}(627 \mathrm{mg}, 2.82 \mathrm{mmol})$, and triethylamine $(0.25 \mathrm{~mL})$ in $\mathrm{CHCl}_{3}(10 \mathrm{~mL})$ at $0{ }^{\circ} \mathrm{C}$ for $3.5 \mathrm{~h}$. Chromatographic purification on silica gel with $\mathrm{CH}_{2} \mathrm{Cl}_{2}$ afforded $\mathbf{2 a}(201 \mathrm{mg}, 59 \%)$. The reaction of $\mathbf{8 a}(109 \mathrm{mg}, 0.386 \mathrm{mmol})$ with Lawesson's reagent (160 mg, $0.396 \mathrm{mmol})$ in toluene $(10 \mathrm{~mL})$ at room temperature for $1.5 \mathrm{~h}$ and chromatographic purification on silica gel with $\mathrm{CH}_{2} \mathrm{Cl}_{2}$ also afforded 2a (64 mg, 56\%). brown green prisms; mp $53.0-55.0{ }^{\circ} \mathrm{C}$; $\mathrm{MS}(70 \mathrm{eV}) \mathrm{m} / \mathrm{z} 298\left(\mathrm{M}^{+}, 91 \%\right)$, $297\left(\mathrm{M}^{+}-\mathrm{H}, 100\right), 295$ (28); IR (KBr disk) $v_{\max } 1388,1278,792,744 \mathrm{~cm}^{-1} ; \mathrm{UV}-\mathrm{vis}\left(\mathrm{CH}_{2} \mathrm{Cl}_{2}\right) \lambda_{\max }, \mathrm{nm}$ (log $\varepsilon) 239$ (4.60), $282 \mathrm{sh}$ (4.44), 295 (4.45), $334 \mathrm{sh}$ (4.23), $350 \mathrm{sh}$ (4.17), 466 (4.32), $590 \mathrm{sh}(3.22) ;{ }^{1} \mathrm{H}$ $\operatorname{NMR}\left(600 \mathrm{MHz}, \mathrm{CDCl}_{3}\right) \delta=9.08\left(\mathrm{~d}, 2 \mathrm{H}, J=9.9 \mathrm{~Hz}, \mathrm{H}_{8}\right), 8.44\left(\mathrm{~d}, 2 \mathrm{H}, J=9.5 \mathrm{~Hz}, \mathrm{H}_{4}\right), 8.13(\mathrm{~d}, 2 \mathrm{H}, J=$ $\left.4.1 \mathrm{~Hz}, \mathrm{H}_{2}\right), 7.70\left(\mathrm{dd}, 2 \mathrm{H}, J=9.8,9.8 \mathrm{~Hz}, \mathrm{H}_{6}\right), 7.42\left(\mathrm{dd}, 2 \mathrm{H}, J=9.8,9.5 \mathrm{~Hz}, \mathrm{H}_{5}\right), 7.33(\mathrm{dd}, 2 \mathrm{H}, J=9.9$, $\left.9.8 \mathrm{~Hz}, \mathrm{H}_{7}\right), 7.29\left(\mathrm{~d}, 2 \mathrm{H}, J=4.1 \mathrm{~Hz}, \mathrm{H}_{3}\right) ;{ }^{13} \mathrm{C} \mathrm{NMR}\left(150 \mathrm{MHz}, \mathrm{CDCl}_{3}\right) \delta=216.1(\mathrm{C}=\mathrm{S}), 146.6\left(\mathrm{C}_{3 \mathrm{a}}\right)$, $141.7\left(\mathrm{C}_{2}\right), 140.4\left(\mathrm{C}_{1}\right), 139.6\left(\mathrm{C}_{6}\right), 139.2\left(\mathrm{C}_{8 \mathrm{a}}\right), 138.7\left(\mathrm{C}_{4}\right), 138.4\left(\mathrm{C}_{8}\right), 128.2\left(\mathrm{C}_{7}\right), 126.9\left(\mathrm{C}_{5}\right), 118.4$ $\left(\mathrm{C}_{3}\right)$. Anal. calcd for $\mathrm{C}_{21} \mathrm{H}_{14} \mathrm{~S} \cdot 1 / 4 \mathrm{H}_{2} \mathrm{O}: \mathrm{C}, 83.27 ; \mathrm{H}, 4.82 ; \mathrm{S}, 10.58$. Found: C, 83.19; H, 4.86; S, 10.35 .

Bis(3,6-di-tert-butyl-1-azulenyl) thioketone (2b). The general procedure was followed by using bis(3,6-di-tert-butyl-1-azulenyl) ketone (8b) (202 mg, $3.99 \mathrm{mmol}), \mathrm{P}_{2} \mathrm{~S}_{5}$ (443 mg, $\left.1.99 \mathrm{mmol}\right)$, and triethylamine $(0.7 \mathrm{~mL})$ in $\mathrm{CHCl}_{3}(15 \mathrm{~mL})$ at room temperature for $2 \mathrm{~h}$. Chromatographic purification on silica gel with $60 \% \mathrm{CH}_{2} \mathrm{Cl}_{2} /$ hexane afforded $\mathbf{2 b}(171 \mathrm{mg}, 82 \%)$. dark green powder; mp $157.8-$ $163.2^{\circ} \mathrm{C}$; MS (70 eV) m/z $522\left(\mathrm{M}^{+}, 100 \%\right), 521(79), 507\left(\mathrm{M}^{+}-\mathrm{CH}_{3}, 20\right), 506(21), 466(23), 465\left(\mathrm{M}^{+}\right.$ - $t$-Bu, 65), 449 (10); IR (KBr disk) $v_{\max } 2963,1576,1418,1374,1227 \mathrm{~cm}^{-1}$; UV-vis $\left(\mathrm{CH}_{2} \mathrm{Cl}_{2}\right) \lambda_{\max }$, nm (log ع) 245 (4.64), 305 (4.54), 353 sh (4.27), 504 (4.42), 610 sh (3.48); ${ }^{1} \mathrm{H}$ NMR (500 MHz, $\left.\mathrm{CDCl}_{3}\right)$ $\delta=9.08\left(\mathrm{~d}, 2 \mathrm{H}, J=10.9 \mathrm{~Hz}, \mathrm{H}_{8}\right), 8.73\left(\mathrm{~d}, 2 \mathrm{H}, J=10.8 \mathrm{~Hz}, \mathrm{H}_{4}\right), 7.93\left(\mathrm{~s}, 2 \mathrm{H}, \mathrm{H}_{2}\right), 7.54(\mathrm{dd}, 2 \mathrm{H}, J=10.8$ $\left.2.0 \mathrm{~Hz}, \mathrm{H}_{5}\right), 7.46\left(\mathrm{dd}, 2 \mathrm{H}, J=10.9,2.0 \mathrm{~Hz}, \mathrm{H}_{7}\right), 1.55(\mathrm{~s}, 18 \mathrm{H}, 3-t-\mathrm{Bu}), 1.44(\mathrm{~s}, 18 \mathrm{H}, 6-t-\mathrm{Bu}) ;{ }^{13} \mathrm{C} \mathrm{NMR}$ $\left(125 \mathrm{MHz}, \mathrm{CDCl}_{3}\right) \delta=212.5(\mathrm{C}=\mathrm{S}), 163.6\left(\mathrm{C}_{6}\right), 141.1\left(\mathrm{C}_{3 \mathrm{a}}\right), 140.2\left(\mathrm{C}_{8 \mathrm{a}}\right), 139.7\left(\mathrm{C}_{2}\right), 138.8\left(\mathrm{C}_{3}\right), 138.1$ 
$\left(\mathrm{C}_{1}\right), 137.2\left(\mathrm{C}_{8}\right), 136.5\left(\mathrm{C}_{4}\right), 126.0\left(\mathrm{C}_{7}\right), 123.4\left(\mathrm{C}_{5}\right), 38.5(\mathrm{~s}, 6-t-\mathrm{Bu}), 33.0(\mathrm{~s}, 3-t-\mathrm{Bu}), 31.8(\mathrm{q}, 3-t-\mathrm{Bu})$, 31.7 (q, 6-t-Bu). Anal. calcd for $\mathrm{C}_{37} \mathrm{H}_{46} \mathrm{~S} \cdot 1 / 4 \mathrm{H}_{2} \mathrm{O}: \mathrm{C}, 84.27 ; \mathrm{H}, 8.89 ; \mathrm{S}, 6.08$. Found: C, 84.40; H, 8.90; S, 5.97.

2-(3,6-Di-tert-butyl-1-azulenyl)-4,7-di-tert-butyl-3H-azuleno[8,1-b,c]thiophene (14b). The reaction of $8 \mathbf{b}(204 \mathrm{mg}, 0.403 \mathrm{mmol})$ with Lawesson's reagent $(207 \mathrm{mg}, 0.512 \mathrm{mmol})$ in toluene $(10 \mathrm{~mL})$ at room temperature for $3 \mathrm{~h}$ and chromatographic purification on $\mathrm{Al}_{2} \mathrm{O}_{3}$ with $40 \% \mathrm{CH}_{2} \mathrm{Cl}_{2} /$ hexane afforded 14b (186 mg, 88\%). brown plates; mp 165.0 - $166 .{ }^{\circ} \mathrm{C}$; MS (70 eV) $\mathrm{m} / z 522\left(\mathrm{M}^{+}, 70 \%\right), 521$ $\left(\mathrm{M}^{+}-\mathrm{H}, 100\right), 465\left(\mathrm{M}^{+}-t\right.$-Bu, 37); IR (KBr disk) $v_{\max } 2964,2868,1576,1464,1366,1242,756 \mathrm{~cm}^{-1}$; UV-vis $\left(\mathrm{CH}_{2} \mathrm{Cl}_{2}\right) \lambda_{\max }, \mathrm{nm}(\log \varepsilon) 240$ (4.41), 254 sh (4.40), 287 (4.43), 329 (4.33), 401 (4.04), 469 sh (3.73), $500 \mathrm{sh}$ (3.71), $543 \mathrm{sh}$ (3.59), $592 \mathrm{sh}$ (3.37), $643 \mathrm{sh}$ (3.19), $686 \mathrm{sh}$ (3.01), $708 \mathrm{sh}(2.85) ;{ }^{1} \mathrm{H}$ NMR $\left(600 \mathrm{MHz}, \mathrm{CDCl}_{3}\right) \delta=8.58\left(\mathrm{~d}, 1 \mathrm{H}, J=10.6 \mathrm{~Hz}, \mathrm{H}_{4^{\prime}}\right), 8.49\left(\mathrm{~d}, 1 \mathrm{H}, J=10.7 \mathrm{~Hz}, \mathrm{H}_{8^{\prime}}\right), 7.84\left(\mathrm{~s}, 1 \mathrm{H}, \mathrm{H}_{2^{\prime}}\right)$, $7.26\left(\mathrm{~d}, 1 \mathrm{H}, J=10.6 \mathrm{~Hz}, \mathrm{H}_{5^{\prime}}\right), 7.26\left(\mathrm{~d}, 1 \mathrm{H}, J=10.7 \mathrm{~Hz}, \mathrm{H}_{7^{\prime}}\right), 6.47$ (d, $\left.1 \mathrm{H}, J=12.9 \mathrm{~Hz}, \mathrm{H}_{5}\right), 6.03(\mathrm{~s}, 1 \mathrm{H}$,

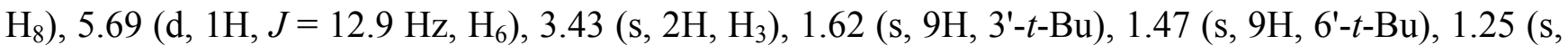
9H, 4-t-Bu), 1.14 (s, 9H, 7-t-Bu); ${ }^{13} \mathrm{C}$ NMR (150 MHz, $\left.\mathrm{CDCl}_{3}\right) \delta=162.1\left(\mathrm{C}_{6}\right), 154.7\left(\mathrm{C}_{8 \mathrm{~b}}\right), 144.4\left(\mathrm{C}_{4}\right)$, $143.4\left(\mathrm{C}_{7}\right), 140.5\left(\mathrm{C}_{2 \mathrm{a}}\right.$ or $\left.\mathrm{C}_{8 \mathrm{a}}\right), 138.9\left(\mathrm{C}_{3^{\prime}}\right), 135.8\left(\mathrm{C}_{3^{\prime} \mathrm{a}}\right), 135.2\left(\mathrm{C}_{4^{\prime}}\right), 134.7\left(\mathrm{C}_{8^{\prime}}\right), 134.4\left(\mathrm{C}_{8^{\prime} \mathrm{a}}\right), 133.9\left(\mathrm{C}_{2^{\prime}}\right)$, $133.1\left(\mathrm{C}_{4 \mathrm{a}}\right), 131.3\left(\mathrm{C}_{2}\right), 128.0\left(\mathrm{C}_{5}\right), 126.9\left(\mathrm{C}_{6}\right), 126.4\left(\mathrm{C}_{2 \mathrm{a}}\right.$ or $\left.\mathrm{C}_{8 \mathrm{a}}\right), 120.9\left(\mathrm{C}_{5^{\prime}}\right.$ or $\left.\mathrm{C}_{7^{\prime}}\right), 120.5\left(\mathrm{C}_{1^{\prime}}\right), 119.9$ $\left(\mathrm{C}_{5^{\prime}}\right.$ or $\left.\mathrm{C}_{7^{\prime}}\right), 119.0\left(\mathrm{C}_{8}\right), 38.3\left(\mathrm{~s}, 6^{\prime}-t-\mathrm{Bu}\right), 36.6(\mathrm{~s}, 7-t-\mathrm{Bu}), 36.0\left(\mathrm{C}_{3}\right), 34.3(\mathrm{~s}, 4-t-\mathrm{Bu}), 33.2\left(\mathrm{~s}, 3^{\prime}-t-\mathrm{Bu}\right)$, 32.0 (q, 3'-t-Bu), 31.7 (q, 6'-t-Bu), 30.2 (q, 4-t-Bu), 29.4 (q, 7-t-Bu). Anal. calcd for $\mathrm{C}_{37} \mathrm{H}_{46} \mathrm{~S} \cdot 3 / 4 \mathrm{H}_{2} \mathrm{O}: \mathrm{C}$, 82.86; H, 8.93; S, 5.98. Found: C, 82.70; H, 8.98; S, 5.93.

Bis(3-methyl-1-azulenyl) thioketone (2c). The general procedure was followed by using bis(3-methyl-1-azulenyl) ketone (8a) (203 mg, $0.654 \mathrm{mmol}), \mathrm{P}_{2} \mathrm{~S}_{5}(729 \mathrm{mg}, 3.28 \mathrm{mmol}$ ), and triethylamine $(1.4 \mathrm{~mL})$ in $\mathrm{CHCl}_{3}(30 \mathrm{~mL})$ at room temperature for $2 \mathrm{~h}$. Chromatographic purification 
on $\mathrm{Al}_{2} \mathrm{O}_{3}$ with $60 \% \mathrm{CH}_{2} \mathrm{Cl}_{2} /$ hexane afforded $2 \mathrm{c}(171 \mathrm{mg}, 80 \%)$. dark green needles; mp $127.0-$ $129.1{ }^{\circ} \mathrm{C}$; MS (70 eV) $\mathrm{m} / z 326\left(\mathrm{M}^{+}, 100 \%\right), 325(44), 311\left(\mathrm{M}^{+}-\mathrm{CH}_{3}, 76\right), 310(26), 295\left(\mathrm{M}^{+}-\mathrm{S}+\mathrm{H}\right.$, 29); IR (KBr disk) $v_{\max } 1406,1389,1370,1192,745,733 \mathrm{~cm}^{-1} ; \mathrm{UV}-\mathrm{vis}\left(\mathrm{CH}_{2} \mathrm{Cl}_{2}\right) \lambda_{\max }, \operatorname{nm}(\log \varepsilon) 242$ (4.65), $286 \mathrm{sh}$ (4.46), 296 (4.47), $335 \mathrm{sh}$ (4.30), $355 \mathrm{sh}$ (4.22), 493 (4.37), $613 \mathrm{sh}(3.53) ;{ }^{1} \mathrm{H}$ NMR (400 $\left.\mathrm{MHz} \mathrm{CDCl}_{3}\right) \delta=8.80\left(\mathrm{~d}, 2 \mathrm{H}, J=10.0 \mathrm{~Hz}, \mathrm{H}_{8}\right), 8.31\left(\mathrm{~d}, 2 \mathrm{H}, J=9.8 \mathrm{~Hz}, \mathrm{H}_{4}\right), 8.03\left(\mathrm{~s}, 2 \mathrm{H}, \mathrm{H}_{2}\right), 7.60(\mathrm{dd}$, $\left.2 \mathrm{H}, J=9.8,9.8 \mathrm{~Hz}, \mathrm{H}_{6}\right), 7.34\left(\mathrm{dd}, 2 \mathrm{H}, J=9.8,9.8 \mathrm{~Hz}, \mathrm{H}_{5}\right), 7.17\left(\mathrm{dd}, 2 \mathrm{H}, J=10.0,9.8 \mathrm{~Hz}, \mathrm{H}_{7}\right), 2.61(\mathrm{~s}$, 6H, 3-Me); ${ }^{13} \mathrm{C}$ NMR (100 MHz, $\left.\mathrm{CDCl}_{3}\right) \delta=214.3(\mathrm{C}=\mathrm{S}), 143.5\left(\mathrm{C}_{3 \mathrm{a}}\right), 142.6\left(\mathrm{C}_{2}\right), 139.3\left(\mathrm{C}_{6}\right), 139.1$ $\left(\mathrm{C}_{8 \mathrm{a}}\right), 138.9\left(\mathrm{C}_{1}\right), 137.8\left(\mathrm{C}_{8}\right), 135.5\left(\mathrm{C}_{4}\right), 127.4\left(\mathrm{C}_{7}\right), 126.6\left(\mathrm{C}_{3}\right), 125.5\left(\mathrm{C}_{5}\right), 12.5(3-\mathrm{Me})$. Anal. calcd for $\mathrm{C}_{23} \mathrm{H}_{18} \mathrm{~S}: \mathrm{C}, 84.62 ; \mathrm{H}, 5.56 ; \mathrm{S}, 9.82$. Found: C, 84.38; H, 5.62; S, 9.53.

2-(3-Methyl-1-azulenyl)-4-methyl-3H-azuleno[8,1-b,c]thiophene (14c). The reaction of $8 \mathrm{c}$ (204 mg, $0.657 \mathrm{mmol})$ with Lawesson's reagent $(336 \mathrm{mg}, 0.831 \mathrm{mmol})$ in toluene $(20 \mathrm{~mL})$ at room temperature for $3 \mathrm{~h}$ and chromatographic purification on $\mathrm{Al}_{2} \mathrm{O}_{3}$ with $40 \%-60 \% \mathrm{CH}_{2} \mathrm{Cl}_{2} /$ hexane afforded $2 \mathrm{c}(27 \mathrm{mg}$, 13\%) and 14c (144 mg, 67\%). dark green needles; mp $131.5-133.0{ }^{\circ} \mathrm{C}$; MS (70 eV) $\mathrm{m} / z 326\left(\mathrm{M}^{+}\right.$, 100\%), 325 (42), $312(20), 311\left(\mathrm{M}^{+}-\mathrm{CH}_{3}, 64\right), 295$ (20); IR (KBr disk) $v_{\max }$ 1565, 1487, 1414, 1364, 733, $681 \mathrm{~cm}^{-1}$; UV-vis $\left(\mathrm{CH}_{2} \mathrm{Cl}_{2}\right) \lambda_{\max }, \mathrm{nm}(\log \varepsilon) 254$ (4.49), 281 (4.47), 322 (4.34), 404 (4.17), 486 (3.63), 521 (3.64), $563 \mathrm{sh}(3.56), 614 \mathrm{sh}(3.39), 684 \mathrm{sh}(3.13) ;{ }^{1} \mathrm{H}$ NMR $\left(600 \mathrm{MHz}, \mathrm{CDCl}_{3}\right) \delta=8.49(\mathrm{~d}$, $\left.1 \mathrm{H}, J=9.8 \mathrm{~Hz}, \mathrm{H}_{8^{\prime}}\right), 8.14\left(\mathrm{~d}, 1 \mathrm{H}, J=9.5 \mathrm{~Hz}, \mathrm{H}_{4^{\prime}}\right), 7.81\left(\mathrm{~s}, 1 \mathrm{H}, \mathrm{H}_{2^{\prime}}\right), 7.51\left(\mathrm{dd}, 1 \mathrm{H}, J=9.8,9.6 \mathrm{~Hz}, \mathrm{H}_{6^{\prime}}\right)$, $7.05\left(\mathrm{dd}, 1 \mathrm{H}, J=9.8,9.6 \mathrm{~Hz}, \mathrm{H}_{7^{\prime}}\right), 7.04\left(\mathrm{dd}, 1 \mathrm{H}, J=9.8,9.5 \mathrm{~Hz}, \mathrm{H}_{5^{\prime}}\right), 5.90\left(\mathrm{~d}, 1 \mathrm{H}, J=11.8 \mathrm{~Hz}, \mathrm{H}_{5}\right)$, $5.83\left(\mathrm{~d}, 1 \mathrm{H}, J=11.4 \mathrm{~Hz}, \mathrm{H}_{8}\right), 5.39\left(\mathrm{dd}, 1 \mathrm{H}, J=11.8,8.1 \mathrm{~Hz}, \mathrm{H}_{6}\right), 5.29\left(\mathrm{dd}, 1 \mathrm{H}, J=11.4,8.1 \mathrm{~Hz}, \mathrm{H}_{7}\right)$, $3.27\left(\mathrm{~s}, 2 \mathrm{H}, \mathrm{H}_{3}\right), 2.63\left(\mathrm{~s}, 3 \mathrm{H}, 3{ }^{\prime}-\mathrm{Me}\right), 1.77(\mathrm{~s}, 3 \mathrm{H}, 4-\mathrm{Me}) ;{ }^{13} \mathrm{C} \mathrm{NMR}\left(150 \mathrm{MHz}, \mathrm{CDCl}_{3}\right) \delta=155.7\left(\mathrm{C}_{8 \mathrm{~b}}\right)$, $141.0\left(\mathrm{C}_{2 \mathrm{a}}\right), 138.8\left(\mathrm{C}_{3^{\prime} \mathrm{a}}\right), 138.5\left(\mathrm{C}_{6^{\prime}}\right), 136.9\left(\mathrm{C}_{2^{\prime}}\right), 135.6\left(\mathrm{C}_{4 \mathrm{a}}\right), 135.5\left(\mathrm{C}_{8^{\prime}}\right), 134.7\left(\mathrm{C}_{8^{\prime} \mathrm{a}}\right), 134.1\left(\mathrm{C}_{4^{\prime}}\right), 132.4$ $\left(\mathrm{C}_{2}\right), 132.2\left(\mathrm{C}_{4}\right), 127.5\left(\mathrm{C}_{5}\right), 126.1\left(\mathrm{C}_{6}\right.$ and $\left.\mathrm{C}_{3^{\prime}}\right), 125.7\left(\mathrm{C}_{8 \mathrm{a}}\right), 125.4\left(\mathrm{C}_{8}\right), 123.3\left(\mathrm{C}_{7}\right), 122.6\left(\mathrm{C}_{5^{\prime}}\right), 122.5$ $\left(\mathrm{C}_{7^{\prime}}\right), 121.2\left(\mathrm{C}_{1^{\prime}}\right), 39.6\left(\mathrm{C}_{3}\right), 13.8(4-\mathrm{Me}), 12.6\left(3^{\prime}-\mathrm{Me}\right)$. Anal. calcd for $\mathrm{C}_{23} \mathrm{H}_{18} \mathrm{~S}: \mathrm{C}, 84.62 ; \mathrm{H}, 5.56 ; \mathrm{S}$, 
9.82. Found: C, 84.35; H, 5.78; S, 9.85.

Bis(6-tert-butyl-1-azulenyl) thioketone (2d). The general procedure was followed by using bis(6-tert-butyl-1-azulenyl) ketone (8d) (208 mg, $0.527 \mathrm{mmol}), \mathrm{P}_{2} \mathrm{~S}_{5}(587 \mathrm{mg}, 2.64 \mathrm{mmol})$, and triethylamine $(1.4 \mathrm{~mL})$ in $\mathrm{CHCl}_{3}(30 \mathrm{~mL})$ at room temperature for $14 \mathrm{~h}$. Chromatographic purification on $\mathrm{Al}_{2} \mathrm{O}_{3}$ with $60 \% \mathrm{CH}_{2} \mathrm{Cl}_{2} /$ hexane afforded $\mathbf{2 d}(133 \mathrm{mg}, 61 \%$ ). The reaction of $\mathbf{8 d}$ (150 $\mathrm{mg}, 0.380$ mmol) with Lawesson's reagent $(161 \mathrm{mg}, 0.398 \mathrm{mmol})$ in toluene $(10 \mathrm{~mL})$ at room temperature for 2.5 $\mathrm{h}$ and chromatographic purification on silica gel with $\mathrm{CH}_{2} \mathrm{Cl}_{2}$ also afforded $\mathbf{2 d}(113 \mathrm{mg}, 72 \%)$. brown green crystals; mp 110.0 - $111.6^{\circ} \mathrm{C}$; MS (70 eV) m/z $410\left(\mathrm{M}^{+}, 100 \%\right), 409(26), 395\left(\mathrm{M}^{+}-\mathrm{CH}_{3}, 30\right)$, 355 (21), 354 (20), $353\left(\mathrm{M}^{+}-t\right.$-Bu, 72); IR (KBr disk) $v_{\max } 1578,1437,1397,1385,1289,795 \mathrm{~cm}^{-1}$; UV-vis $\left(\mathrm{CH}_{2} \mathrm{Cl}_{2}\right) \lambda_{\max }, \mathrm{nm}(\log \varepsilon) 241$ (4.64), 284 sh (4.48), 301 (4.57), 340 sh (4.33), 357 sh (4.29), 476 (4.47), $584 \mathrm{sh}(3.43) ;{ }^{1} \mathrm{H}$ NMR (400 MHz, $\left.\mathrm{CDCl}_{3}\right) \delta=9.19\left(\mathrm{~d}, 2 \mathrm{H}, J=10.5 \mathrm{~Hz}, \mathrm{H}_{8}\right), 8.38(\mathrm{~d}, 2 \mathrm{H}, J$ $\left.=10.3 \mathrm{~Hz}, \mathrm{H}_{4}\right), 8.00\left(\mathrm{~d}, 2 \mathrm{H}, J=4.2 \mathrm{~Hz}, \mathrm{H}_{2}\right), 7.60\left(\mathrm{dd}, 2 \mathrm{H}, J=10.3,1.8 \mathrm{~Hz}, \mathrm{H}_{5}\right), 7.57(\mathrm{dd}, 2 \mathrm{H}, J=10.5$, $\left.1.8 \mathrm{~Hz}, \mathrm{H}_{7}\right), 7.19\left(\mathrm{~d}, 2 \mathrm{H}, J=4.2 \mathrm{~Hz}, \mathrm{H}_{3}\right), 1.44(\mathrm{~s}, 18 \mathrm{H}, 6-t-\mathrm{Bu}) ;{ }^{13} \mathrm{C} \mathrm{NMR}\left(100 \mathrm{MHz}, \mathrm{CDCl}_{3}\right) \delta=214.8$ $(\mathrm{C}=\mathrm{S}), 164.2\left(\mathrm{C}_{6}\right), 145.5\left(\mathrm{C}_{3 \mathrm{a}}\right), 140.9\left(\mathrm{C}_{2}\right), 140.2\left(\mathrm{C}_{1}\right), 138.8\left(\mathrm{C}_{8 \mathrm{a}}\right), 137.7\left(\mathrm{C}_{8}\right), 137.6\left(\mathrm{C}_{4}\right), 126.6\left(\mathrm{C}_{7}\right)$, $125.2\left(\mathrm{C}_{5}\right), 117.7\left(\mathrm{C}_{3}\right), 38.8(\mathrm{~s}, 6-t-\mathrm{Bu}), 31.8(\mathrm{q}, 6-t-\mathrm{Bu})$. Anal. calcd for $\mathrm{C}_{29} \mathrm{H}_{30} \mathrm{~S} \cdot \mathrm{H}_{2} \mathrm{O}: \mathrm{C}, 81.26 ; \mathrm{H}$, 7.52; S, 7.48. Found: C, 81.02; H, 7.62; S, 7.20.

2-Phenyl-4,7-di-tert-butylazuleno[8,1-b,c]thienyl hexafluophosphate $\left(\mathbf{1 8}^{+} \cdot \mathbf{P F}_{\mathbf{6}}{ }^{\top}\right)$. DDQ (148 $\mathrm{mg}$, $0.652 \mathrm{mmol})$ was added at room temperature to a solution of $13 \mathbf{b}(187 \mathrm{mg}, 0.519 \mathrm{mmol})$ in $\mathrm{CH}_{2} \mathrm{Cl}_{2}(40$ $\mathrm{mL}$ ). The solution was stirred at room temperature for 30 min until the reaction was completed. A $60 \%$ aqueous $\mathrm{HPF}_{6}$ solution $(5 \mathrm{~mL})$ was added to the reaction mixture. After stirring at room temperature for an additional $10 \mathrm{~min}$, water was added to the mixture. The resulting suspension was filtered with suction. The organic layer was separated, washed with water, dried over $\mathrm{MgSO}_{4}$, and concentrated 
under reduced pressure. The residue was dissolved in small amount of $\mathrm{CH}_{2} \mathrm{Cl}_{2}$ and then poured into ether. The precipitated crystals were collected by filtration, washed with ether, and dried in vacuo to give $18^{+} \cdot \mathrm{PF}_{6}^{-}(250 \mathrm{mg}, 96 \%)$. reddish brown crystals; mp $267.7-269.5^{\circ} \mathrm{C}$; MS (FAB) $m / z 359\left(\mathrm{M}^{+}-\right.$ $\left.\mathrm{PF}_{6}\right) ; \mathrm{IR}\left(\mathrm{KBr}\right.$ disk) $v_{\max } 1385,841\left(\mathrm{~s}, \mathrm{PF}_{6}{ }^{-}\right), 558\left(\mathrm{~m}, \mathrm{PF}_{6}{ }^{-}\right) \mathrm{cm}^{-1} ; \mathrm{UV}-\mathrm{vis}(\mathrm{MeCN}) \lambda_{\max }, \mathrm{nm}(\log \varepsilon) 257$ (4.29), 279 (4.32), 313 (4.27), $377 \mathrm{sh}$ (4.10), 413 (4.19), 546 (3.43); ${ }^{1} \mathrm{H}$ NMR (600 MHz, $\left.\mathrm{CF}_{3} \mathrm{CO}_{2} \mathrm{D}\right) \delta$ $=9.24\left(\mathrm{~d}, 1 \mathrm{H}, J=10.6 \mathrm{~Hz}, \mathrm{H}_{5}\right), 9.23\left(\mathrm{~s}, 1 \mathrm{H}, \mathrm{H}_{8}\right), 9.11\left(\mathrm{~d}, 1 \mathrm{H}, J=10.6 \mathrm{~Hz}, \mathrm{H}_{6}\right), 8.04(\mathrm{~d}, 2 \mathrm{H}, J=7.7 \mathrm{~Hz}$, $\left.\mathrm{H}_{2^{\prime}, 6^{\prime}}\right), 7.78\left(\mathrm{~s}, 1 \mathrm{H}, \mathrm{H}_{3}\right), 7.70\left(\mathrm{t}, 1 \mathrm{H}, J=7.5 \mathrm{~Hz}, \mathrm{H}_{4^{\prime}}\right), 7.62\left(\mathrm{dd}, 2 \mathrm{H}, J=7.7,7.5 \mathrm{~Hz}, \mathrm{H}_{3^{\prime}, 5^{\prime}}\right), 1.65(\mathrm{~s}, 9 \mathrm{H}$, 7-t-Bu), $1.60(\mathrm{~s}, 9 \mathrm{H}, 4-t-\mathrm{Bu}) ;{ }^{13} \mathrm{C} \mathrm{NMR}\left(150 \mathrm{MHz}, \mathrm{CF}_{3} \mathrm{CO}_{2} \mathrm{D}\right) \delta=170.8\left(\mathrm{C}_{7}\right), 169.1\left(\mathrm{C}_{2}\right.$ or $\left.\mathrm{C}_{2 \mathrm{a}}\right), 164.1$ $\left(\mathrm{C}_{4}\right), 154.7\left(\mathrm{C}_{4 \mathrm{a}}\right), 154.2\left(\mathrm{C}_{8 \mathrm{~b}}\right), 152.8\left(\mathrm{C}_{8 \mathrm{a}}\right), 145.8\left(\mathrm{C}_{6}\right), 141.0\left(\mathrm{C}_{5}\right), 140.9\left(\mathrm{C}_{2}\right.$ or $\left.\mathrm{C}_{2 \mathrm{a}}\right), 140.5\left(\mathrm{C}_{8}\right), 136.9$ $\left(\mathrm{C}_{4^{\prime}}\right), 133.7\left(\mathrm{C}_{3}\right), 132.8\left(\mathrm{C}_{1^{\prime}}\right), 132.0\left(\mathrm{C}_{3^{\prime}, 5^{\prime}}\right), 131.8\left(\mathrm{C}_{2^{\prime}, 6^{\prime}}\right), 42.5(\mathrm{~s}, 7-t-\mathrm{Bu}), 36.1(\mathrm{~s}, 4-t-\mathrm{Bu}), 32.4(\mathrm{q}$, 7-t-Bu), 31.2 (q, 4-t-Bu). Anal. calcd for $\mathrm{C}_{25} \mathrm{H}_{27} \mathrm{SPF}_{6}: \mathrm{C}, 59.52 ; \mathrm{H}, 5.39 ; \mathrm{S}, 6.36$. Found: C, 59.74; $\mathrm{H}$, $5.35 ; \mathrm{S}, 6.90$.

\section{2-(3,6-Di-tert-butyl-1-azulenyl)-4,7-di-tert-butylazuleno[8,1-b,c]thienyl}

$\left(\mathbf{1 9}^{+} \cdot \mathbf{P F}_{\mathbf{6}}{ }^{-}\right)$. The same procedure used for the preparation of $18^{+} \cdot \mathrm{PF}_{6}^{-}$was adopted. The reaction of $\mathbf{1 4 b}$ (143 mg, $0.274 \mathrm{mmol})$ with DDQ $(74 \mathrm{mg}, 0.33 \mathrm{mmol})$ in $\mathrm{CH}_{2} \mathrm{Cl}_{2}(20 \mathrm{~mL})$ at room temperature for 30 min and crystallization from $\mathrm{CH}_{2} \mathrm{Cl}_{2}$-ether afforded $19^{+} \cdot \mathrm{PF}_{6}^{-}(123 \mathrm{mg}, 67 \%)$. deep blue crystals; mp 267.8 - 269.1 ${ }^{\circ} \mathrm{C}$; MS (FAB) $m / z 521\left(\mathrm{M}^{+}-\mathrm{PF}_{6}\right)$; IR (KBr disk) $v_{\max } 1499,1485,1428,1368,1341$, 1298, 1242, 1111, $841\left(\mathrm{~s}, \mathrm{PF}_{6}{ }^{-}\right), 558\left(\mathrm{~m}, \mathrm{PF}_{6}{ }^{-}\right) \mathrm{cm}^{-1}$; UV-vis $(\mathrm{MeCN}) \lambda_{\max }, \mathrm{nm}(\log \varepsilon) 244(4.59), 269$ (4.67), 303 (4.38), $321 \mathrm{sh}$ (4.30), $351 \mathrm{sh}$ (4.09), 414 (3.96), $538 \mathrm{sh}$ (4.18), 639 (4.56), $554 \mathrm{sh}(4.54) ;{ }^{1} \mathrm{H}$ $\operatorname{NMR}\left(600 \mathrm{MHz}, \mathrm{CDCl}_{3}\right) \delta=9.37\left(\mathrm{~d}, 1 \mathrm{H}, J=10.7 \mathrm{~Hz}, \mathrm{H}_{8}\right), 8.95\left(\mathrm{~d}, 1 \mathrm{H}, J=10.5 \mathrm{~Hz}, \mathrm{H}_{5}\right), 8.92(\mathrm{~d}, 1 \mathrm{H}$, $\left.J=10.8 \mathrm{~Hz}, \mathrm{H}_{4}{ }^{\prime}\right), 8.69\left(\mathrm{~d}, 1 \mathrm{H}, J=1.4 \mathrm{~Hz}, \mathrm{H}_{8}\right), 8.47\left(\mathrm{dd}, 1 \mathrm{H}, J=10.5,1.4 \mathrm{~Hz}, \mathrm{H}_{6}\right), 8.27\left(\mathrm{~s}, 1 \mathrm{H}, \mathrm{H}_{2}{ }^{\prime}\right)$, $8.25\left(\mathrm{dd}, 1 \mathrm{H}, J=10.7,1.9 \mathrm{~Hz}, \mathrm{H}_{7}\right), 8.07\left(\mathrm{dd}, 1 \mathrm{H}, J=10.8,1.9 \mathrm{~Hz}, \mathrm{H}_{5}\right), 7.76\left(\mathrm{~s}, 1 \mathrm{H}, \mathrm{H}_{3}\right), 1.66(\mathrm{~s}, 9 \mathrm{H}$, $\left.3^{\prime}-t-\mathrm{Bu}\right), 1.63(\mathrm{~s}, 9 \mathrm{H}, 4-t-\mathrm{Bu}), 1.61(\mathrm{~s}, 9 \mathrm{H}, 7-t-\mathrm{Bu}), 1.55\left(\mathrm{~s}, 9 \mathrm{H}, 66^{\prime}-t-\mathrm{Bu}\right) ;{ }^{13} \mathrm{C} \mathrm{NMR}\left(150 \mathrm{MHz}, \mathrm{CDCl}_{3}\right) \delta$ 
$=168.6\left(\mathrm{C}_{6^{\prime}}\right), 166.1\left(\mathrm{C}_{7}\right), 157.4\left(\mathrm{C}_{2}\right.$ or $\left.\mathrm{C}_{2 \mathrm{a}}\right), 156.1\left(\mathrm{C}_{4}\right), 150.9\left(\mathrm{C}_{8 \mathrm{a}}\right), 148.7\left(\mathrm{C}_{8 \mathrm{~b}}\right), 147.1\left(\mathrm{C}_{3^{\prime} \mathrm{a}}\right), 146.7$ $\left(\mathrm{C}_{3^{\prime}}\right), 145.3\left(\mathrm{C}_{8^{\prime}}\right), 144.1\left(\mathrm{C}_{4 \mathrm{a}}\right), 138.5\left(\mathrm{C}_{5}\right.$ or $\left.\mathrm{C}_{4^{\prime}}\right), 138.4\left(\mathrm{C}_{5}\right.$ or $\left.\mathrm{C}_{4^{\prime}}\right), 137.5\left(\mathrm{C}_{8^{\prime}}\right), 136.9\left(\mathrm{C}_{2}{ }^{\prime}\right), 136.1\left(\mathrm{C}_{2}\right.$ or $\left.\mathrm{C}_{2 \mathrm{a}}\right), 135.8\left(\mathrm{C}_{6}\right), 132.6\left(\mathrm{C}_{7}\right), 131.5\left(\mathrm{C}_{8}\right), 131.4\left(\mathrm{C}_{5}\right), 131.3\left(\mathrm{C}_{3}\right), 122.7\left(\mathrm{C}_{1}\right), 40.5(\mathrm{~s}, 7-t-\mathrm{Bu}), 39.4(\mathrm{~s}$, 6'-t-Bu), 34.3 (s, 4-t-Bu), 33.2 (s, 3'-t-Bu), 31.9 (q, 7-t-Bu), 31.6 (q, 6'-t-Bu), 31.3 (q, 4-t-Bu), 31.0 (q, 3'-t-Bu). Anal. calcd for $\mathrm{C}_{37} \mathrm{H}_{45} \mathrm{SPF}_{6} \cdot 1 / 2 \mathrm{H}_{2} \mathrm{O}: \mathrm{C}, 65.76 ; \mathrm{H}, 6.86 ; \mathrm{S}, 4.74$. Found: $\mathrm{C}, 65.89 ; \mathrm{H}, 6.66 ; \mathrm{S}$, 4.77 . 


\section{Cyclic Voltammograms}

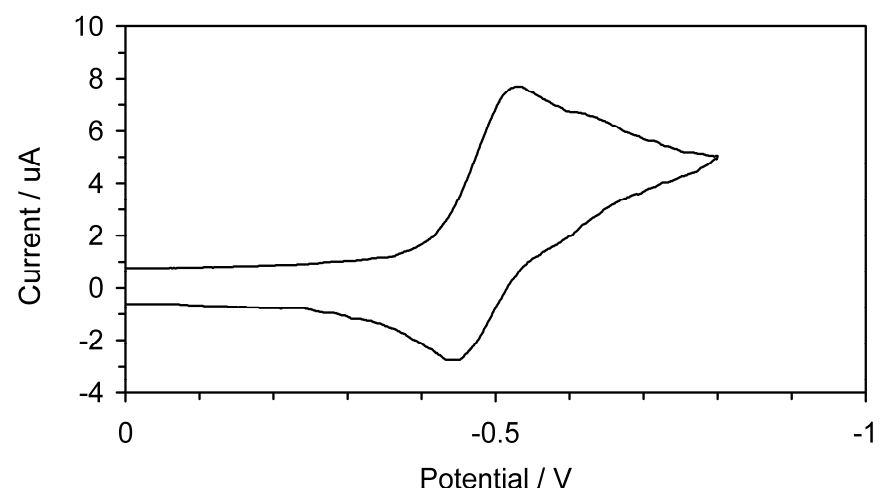

Figure S-1. Reduction wave upon $\mathrm{CV}$ of $\mathbf{1 8}^{+} \cdot \mathrm{PF}_{6}{ }^{-}(1 \mathrm{mM})$ in acetonitrile containing $\mathrm{Et}_{4} \mathrm{NClO}_{4}(0.1 \mathrm{M})$ as a supporting electrolyte.

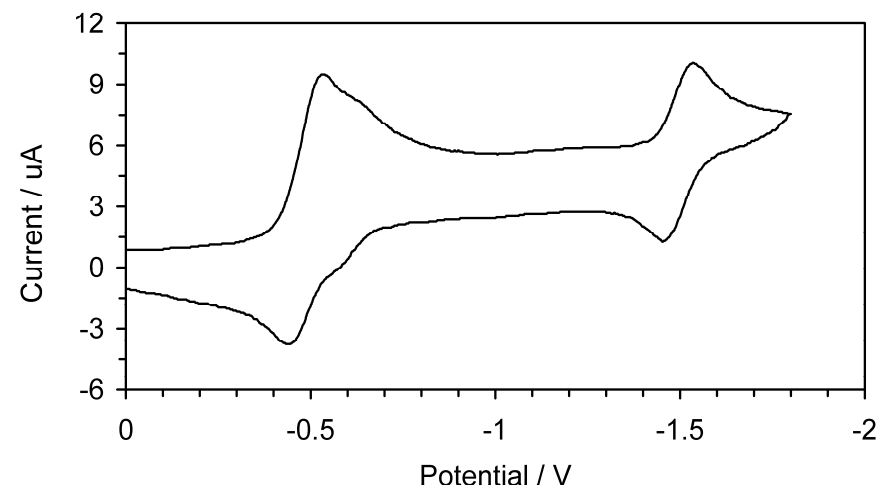

Figure S-2. Reduction wave upon $\mathrm{CV}$ of $\mathbf{1 8}^{+} \cdot \mathrm{PF}_{6}{ }^{-}(1 \mathrm{mM})$ in acetonitrile containing $\mathrm{Et}_{4} \mathrm{NClO}_{4}(0.1 \mathrm{M})$ as a supporting electrolyte. 


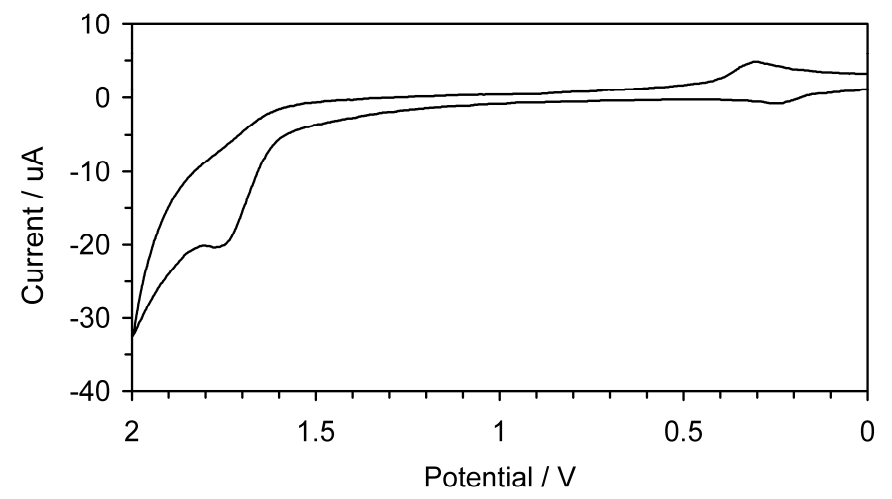

Figure S-3. Oxidation wave upon $\mathrm{CV}$ of $\mathbf{1 8}^{+} \cdot \mathrm{PF}_{6}{ }^{-}(1 \mathrm{mM})$ in acetonitrile containing $\mathrm{Et}_{4} \mathrm{NClO}_{4}(0.1 \mathrm{M})$ as a supporting electrolyte.

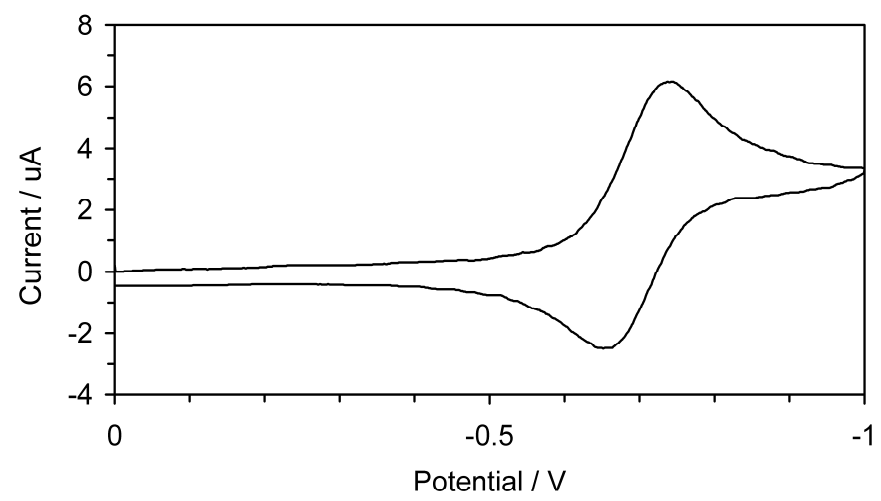

Figure S-4. Reduction wave upon $\mathrm{CV}$ of $\mathbf{1 9}^{+} \cdot \mathrm{PF}_{6}{ }^{-}(1 \mathrm{mM})$ in acetonitrile containing $\mathrm{Et}_{4} \mathrm{NClO}_{4}(0.1 \mathrm{M})$ as a supporting electrolyte. 


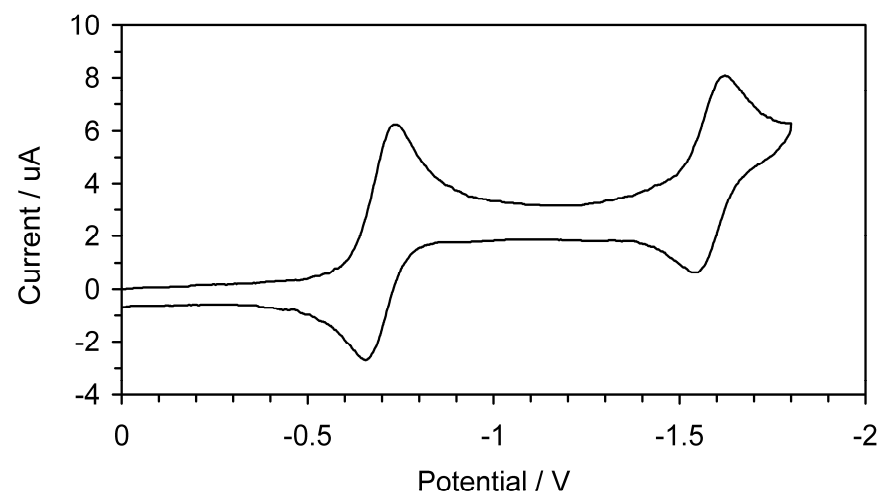

Figure S-5. Reduction wave upon $\mathrm{CV}$ of $\mathbf{1 9}^{+} \cdot \mathrm{PF}_{6}^{-}(1 \mathrm{mM})$ in acetonitrile containing $\mathrm{Et}_{4} \mathrm{NClO}_{4}(0.1 \mathrm{M})$ as a supporting electrolyte.

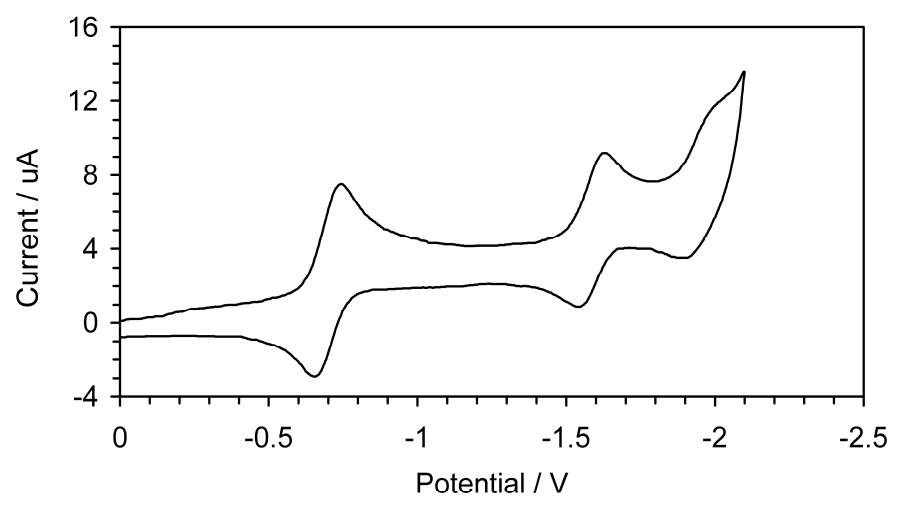

Figure S-6. Reduction wave upon $\mathrm{CV}$ of $\mathbf{1 9}^{+} \cdot \mathrm{PF}_{6}{ }^{-}(1 \mathrm{mM})$ in acetonitrile containing $\mathrm{Et}_{4} \mathrm{NClO}_{4}(0.1 \mathrm{M})$ as a supporting electrolyte. 


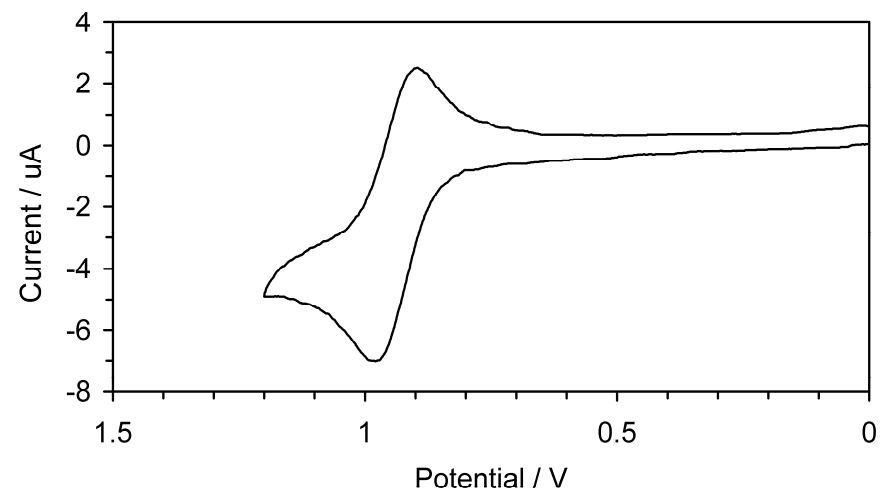

Figure S-7. Oxidation wave upon $\mathrm{CV}$ of $\mathbf{1 9}^{+} \cdot \mathrm{PF}_{6}{ }^{-}(1 \mathrm{mM})$ in acetonitrile containing $\mathrm{Et}_{4} \mathrm{NClO}_{4}(0.1 \mathrm{M})$ as a supporting electrolyte.

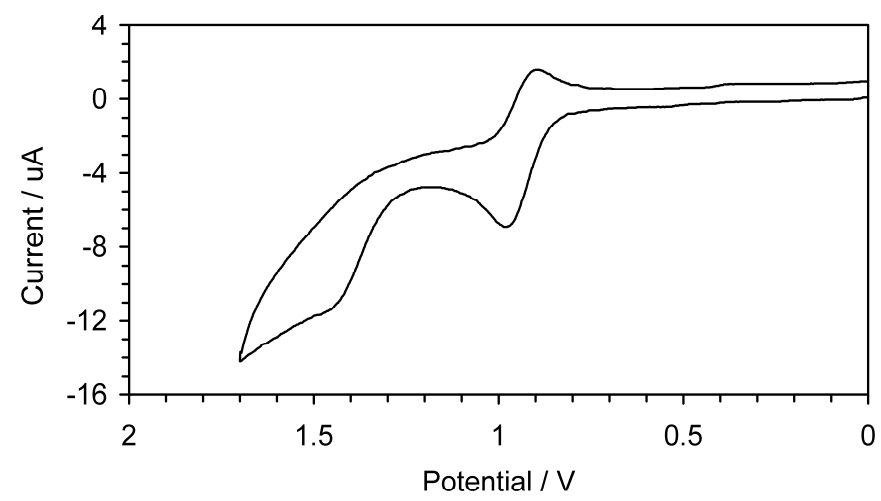

Figure S-8. Oxidation wave upon $\mathrm{CV}$ of $\mathbf{1 9}^{+} \cdot \mathrm{PF}_{6}^{-}(1 \mathrm{mM})$ in acetonitrile containing $\mathrm{Et}_{4} \mathrm{NClO}_{4}(0.1 \mathrm{M})$ as a supporting electrolyte. 


\section{Spectroelectrograms}

(a)

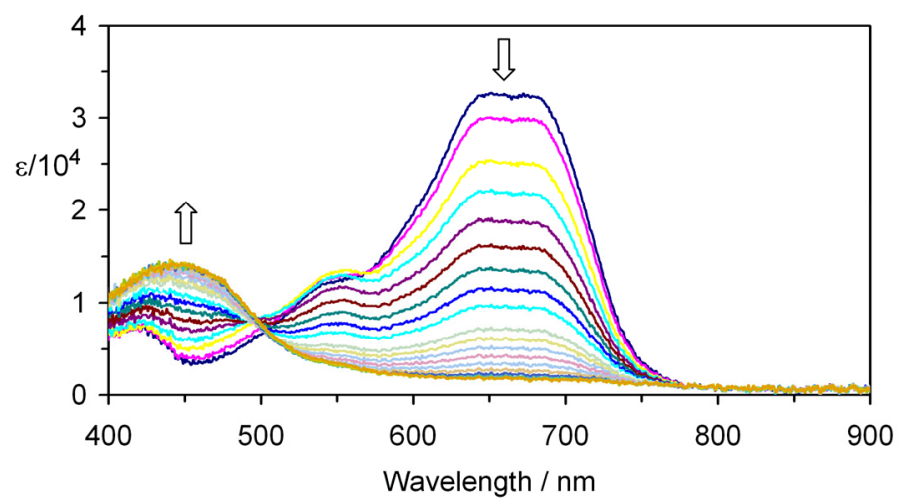

(b)

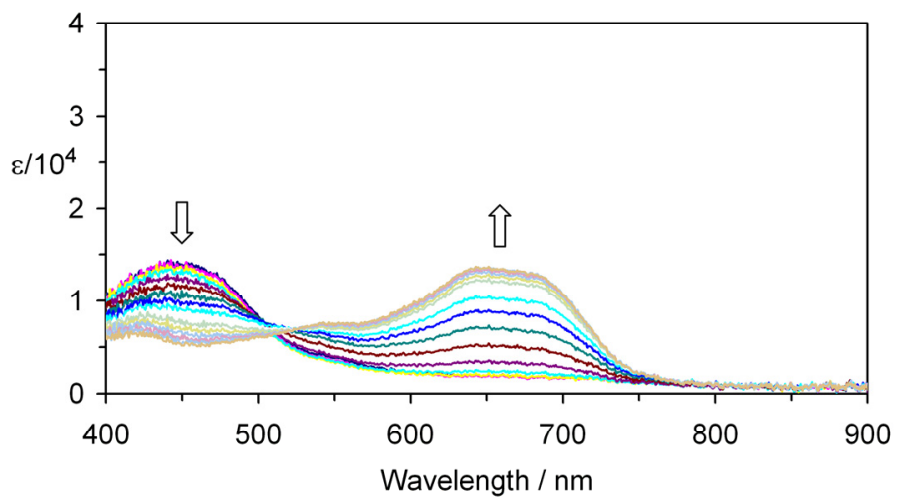

(c)

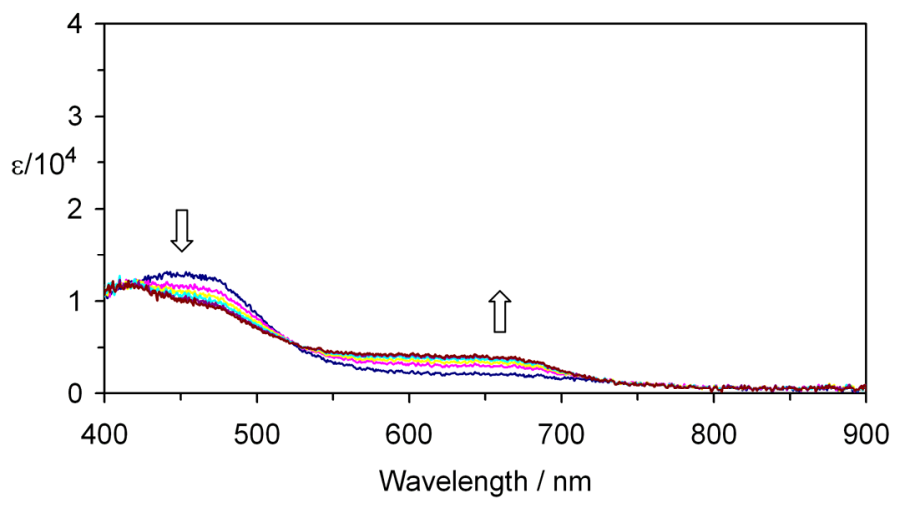

Figure S-9. Continuous change in visible spectra of $\mathbf{1 9}^{+} \cdot \mathrm{PF}_{6}^{-}$: (a) constant-current electrochemical reduction (50 uA, $30 \mathrm{sec}$ intervals), (b) reverse oxidation of the yellow colored species (50 uA, $30 \mathrm{sec}$ intervals), and (c) further reduction of the yellow colored species (100 uA, 5 min intervals) in benzonitrile $\left(2 \mathrm{~mL} ; 1.9 \times 10^{-4} \mathrm{M}\right)$ containing $\mathrm{Et}_{4} \mathrm{NClO}_{4}(0.1 \mathrm{M})$. 
(a)

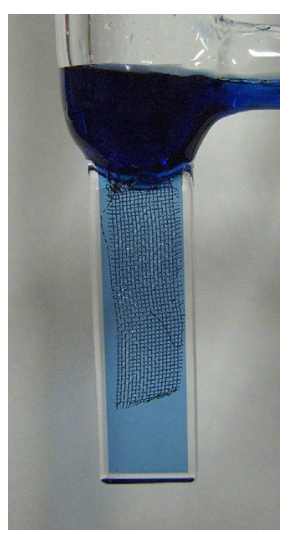

(b)

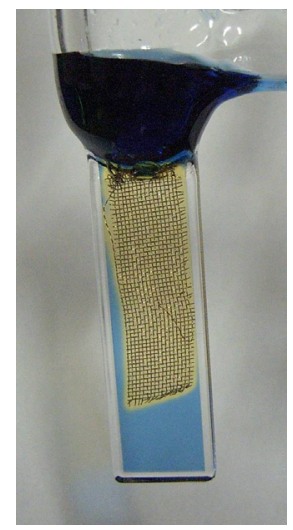

Figure S-10. Color changes of $\mathbf{1 9}^{+} \cdot \mathrm{PF}_{6}{ }^{-}$upon the electrochromic analysis in benzonitrile $(2 \mathrm{~mL} ; 1.9 \times$ $\left.10^{-4} \mathrm{M}\right)$ containing $\mathrm{Et}_{4} \mathrm{NClO}_{4}(0.1 \mathrm{M})$ upon $(50 \mathrm{uA})$ : (a) before electrochemical reduction and (b) after electrochemical reduction for $1 \mathrm{~h}$. 


\section{ESR measurements}

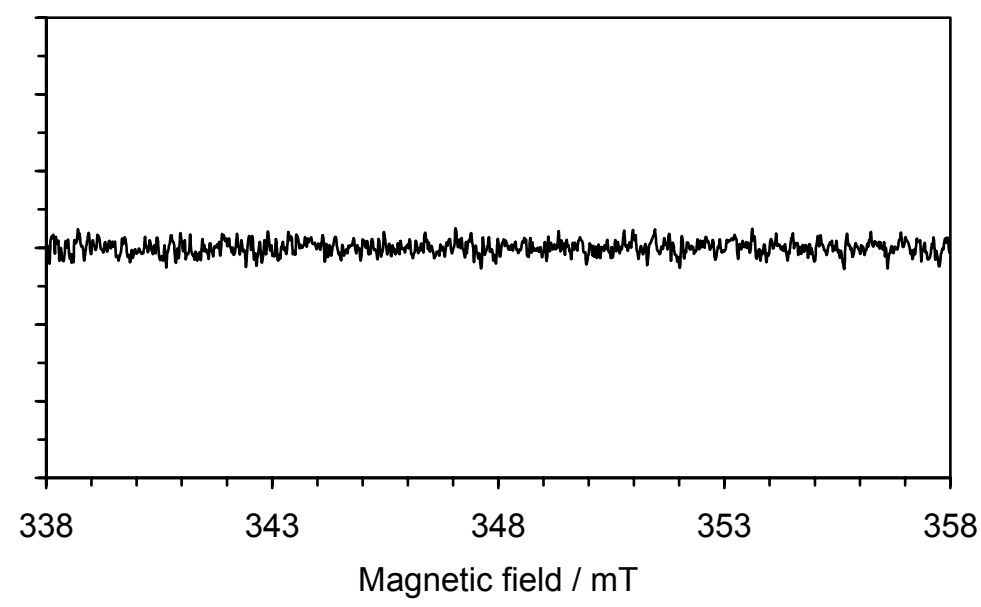

Figure S-11. ESR spectrum of $\mathbf{1 9}^{+} \cdot \mathrm{PF}_{6}{ }^{-}$(before electrochemical reduction).

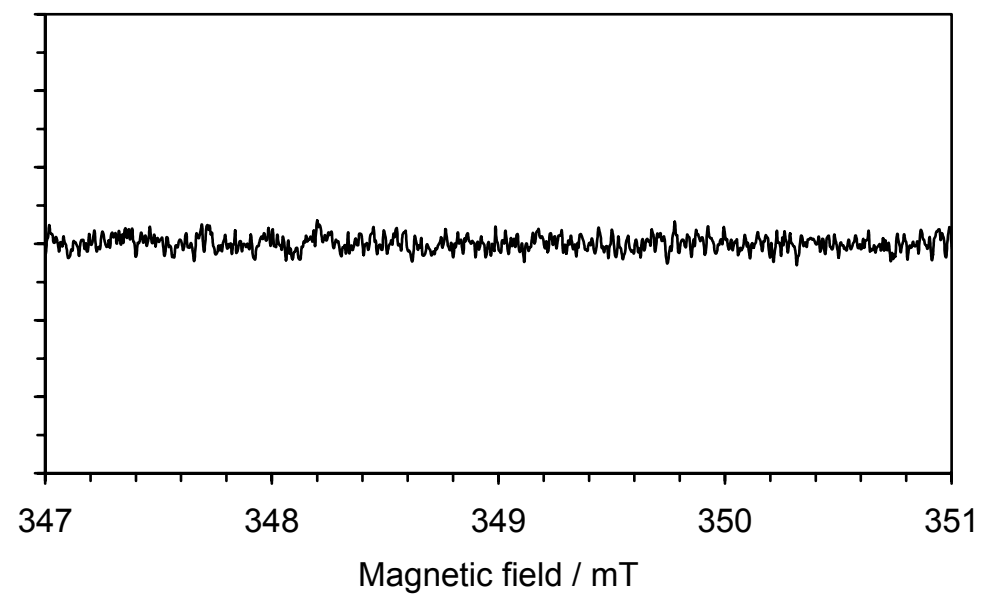

Figure S-12. ESR spectrum of the yellow colored species generated by the electrochemical reduction of $19^{+} \cdot \mathrm{PF}_{6}{ }^{-}$at $-2.0 \mathrm{~V}$ for $2 \mathrm{~h}$ at room temperature. 
(a)

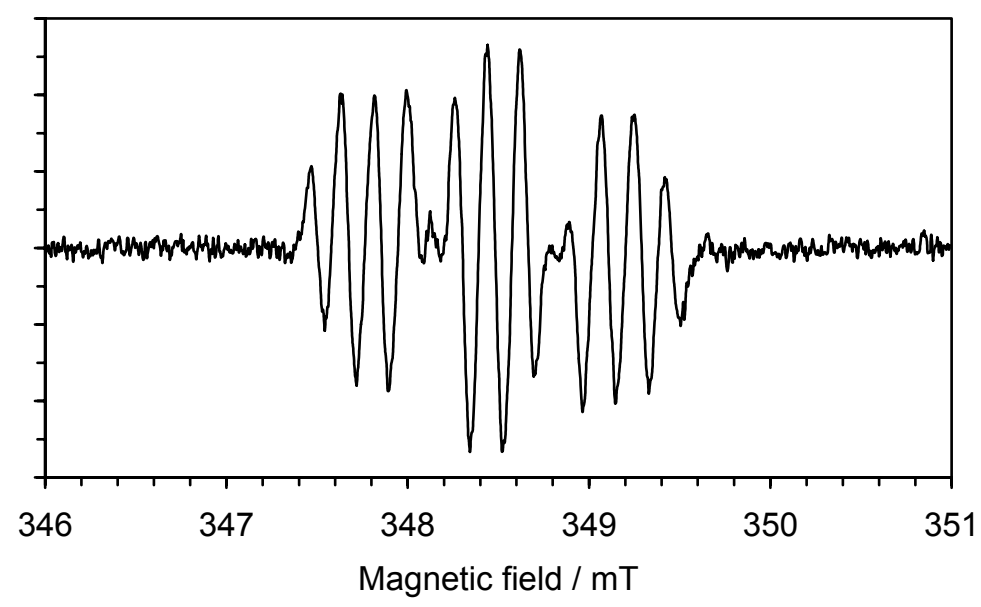

(b)

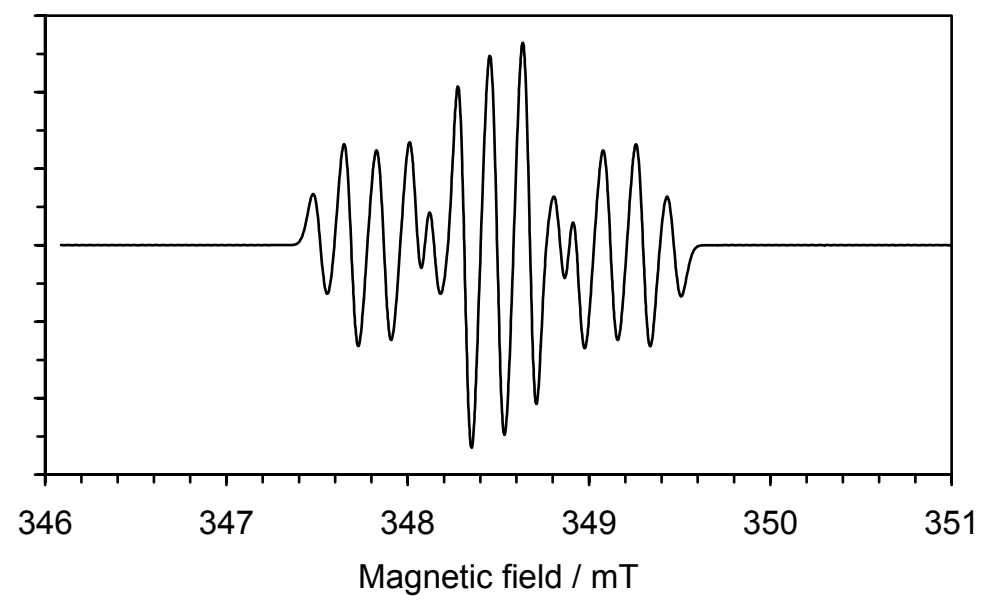

Figure S-13. ESR spectrum of the reddish-brown colored species generated by the electrochemical reduction of $19^{+} \cdot \mathrm{PF}_{6}^{-}$at $-3.0 \mathrm{~V}$ for $2 \mathrm{~h}$ at room temperature: (a) observed ESR spectrum and (b) computer simulation. 
(a)

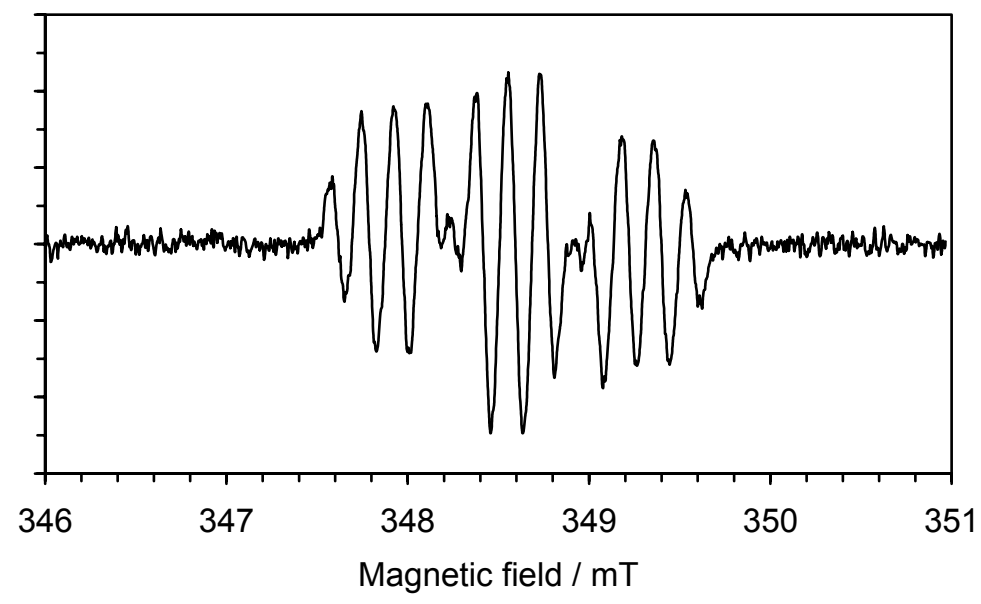

(b)

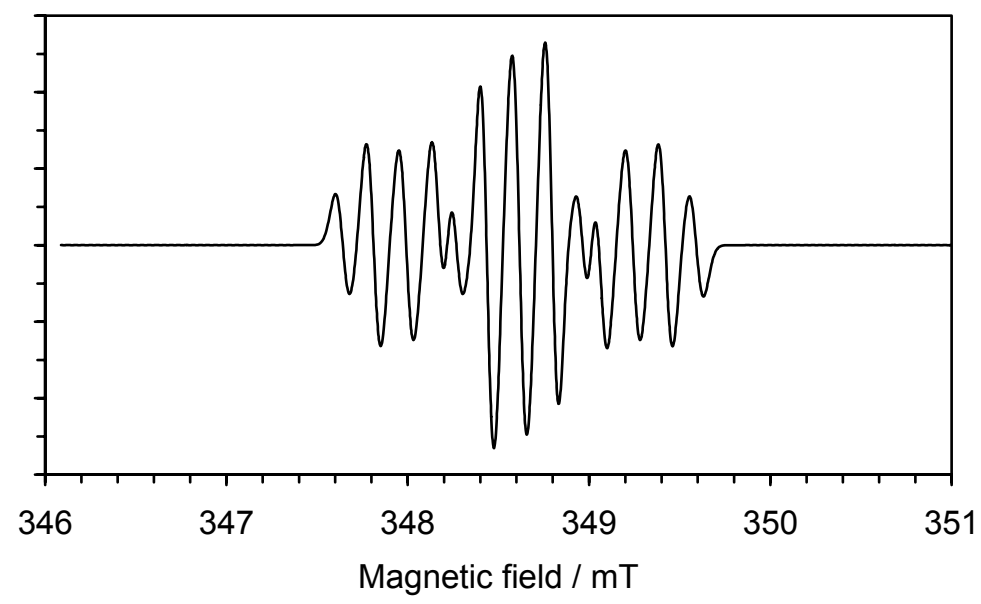

Figure S-14. ESR spectrum of the reddish-brown colored species generated by the electrochemical reduction of $19^{+} \cdot \mathrm{PF}_{6}{ }^{-}$at $-5.0 \mathrm{~V}$ for $14 \mathrm{~h}$ at room temperature: (a) observed ESR spectrum and (b) computer simulation. 
(a)

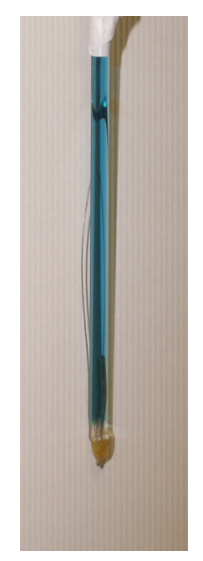

(b)

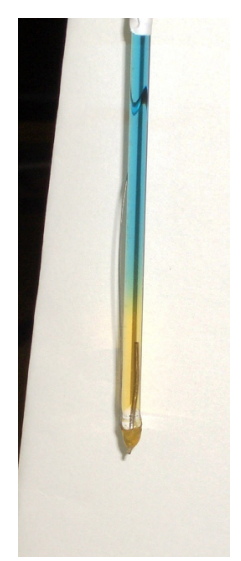

(c)

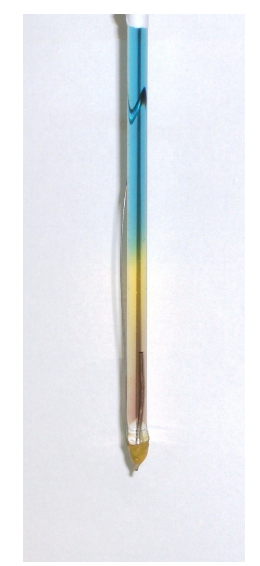

(d)

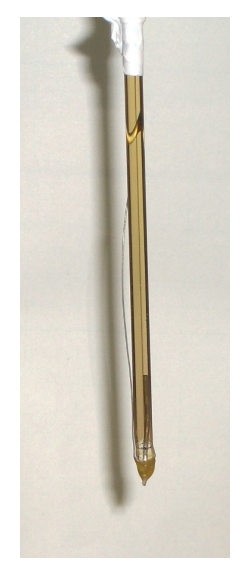

Figure S-15. Color changes of the ESR sample of $\mathbf{1 9}^{+} \cdot \mathrm{PF}_{6}^{-}$in dimethylformamide containing $\mathrm{Et}_{4} \mathrm{NClO}_{4}$ upon electrochemical reduction: (a) before electrochemical reduction, (b) after electrochemical reduction at $-2.0 \mathrm{~V}$ for $2 \mathrm{~h}$, (c) after electrochemical reduction at $-3.0 \mathrm{~V}$ for $2 \mathrm{~h}$, and (d) after electrochemical reduction at $-5.0 \mathrm{~V}$ for $14 \mathrm{~h}$.

Table S-1. ESR Parameters for the Reddish-brown Colored Reduction Product of $19^{+} \cdot \mathrm{PF}_{6}^{-}$

\begin{tabular}{ccc}
\hline Parameter & Element & Value \\
\hline$g$ & & 2.00235 \\
$a_{1}$ & $\mathrm{H}_{4}, \mathrm{H}_{8}$ & $0.625 \mathrm{mT}$ \\
$a_{2}$ & $\mathrm{H}_{2}$ & $0.360 \mathrm{mT}$ \\
$a_{3}$ & $\mathrm{H}_{5}, \mathrm{H}_{7}$ & $0.170 \mathrm{mT}$ \\
$\Gamma$ & Gaussian $(\mathrm{HWHM})$ & $0.080 \mathrm{mT}$ \\
\hline
\end{tabular}




\section{B3LYP/6-31G ${ }^{* *}$ density functional calculation of 1-azulenecarbothialdehyde}<smiles>S=Cc1ccc2cccccc1-2</smiles>

Cartesian Coordinates

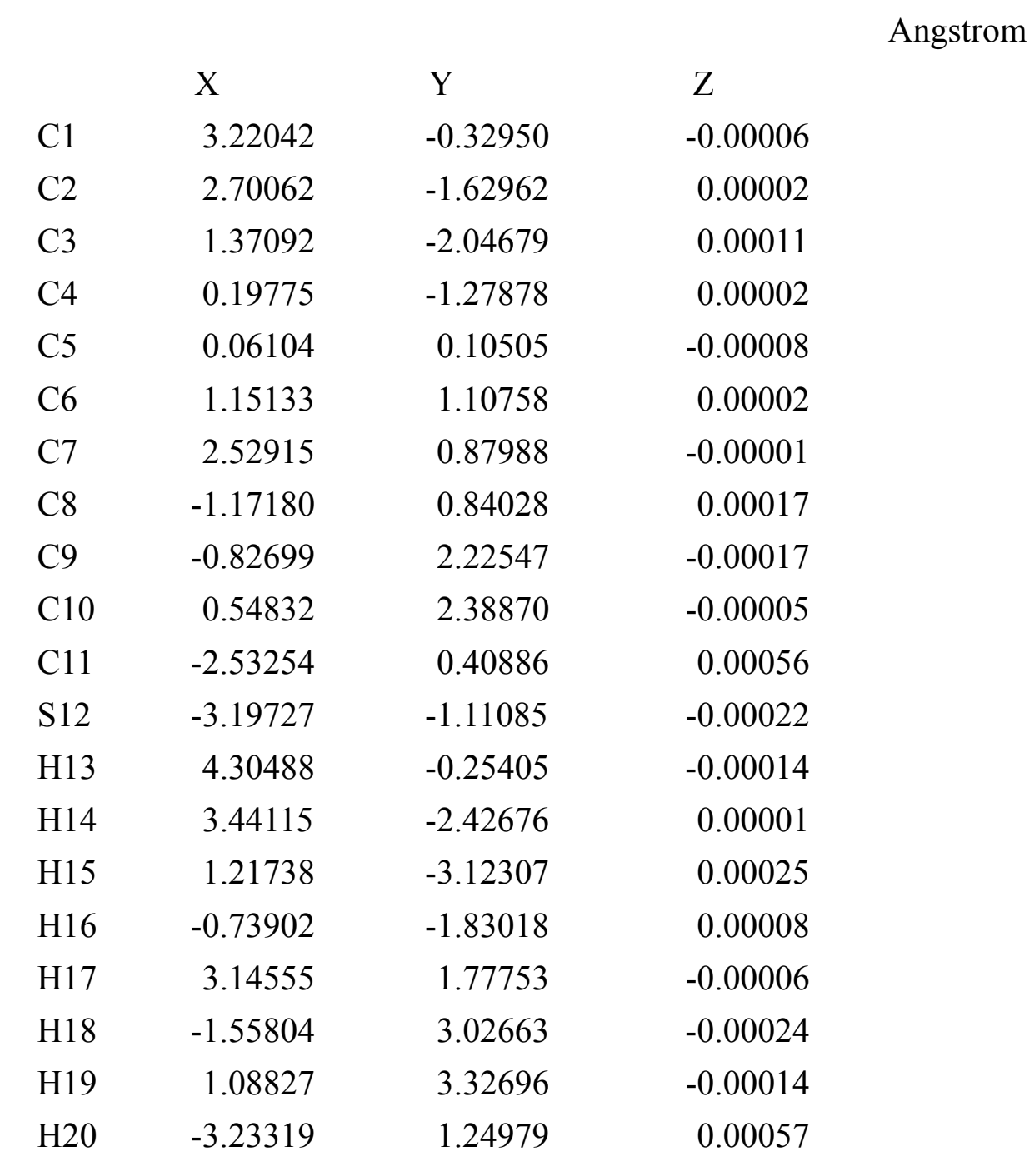


$\mathrm{LUMO}+3(+0.066112 \mathrm{au})$

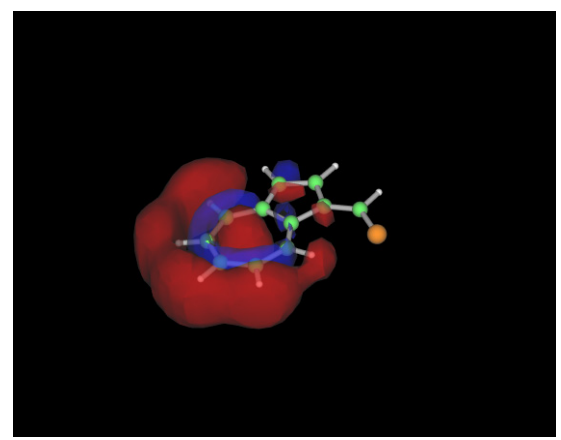

$\mathrm{LUMO}+2(-0.022399 \mathrm{au})$

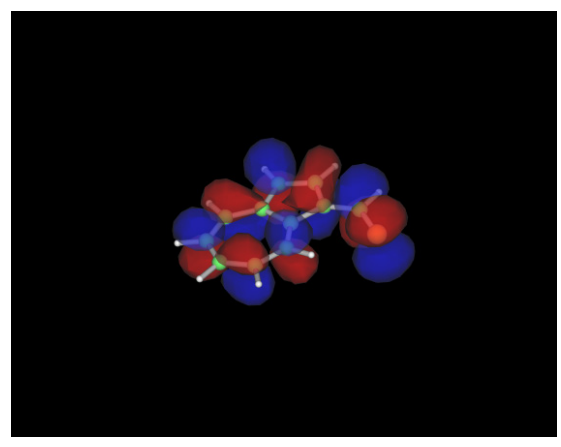

LUMO+1 (-0.088287 au)

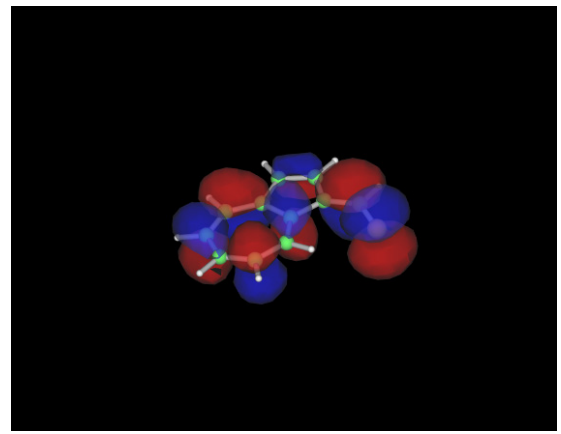

LUMO (-0.089801 au)

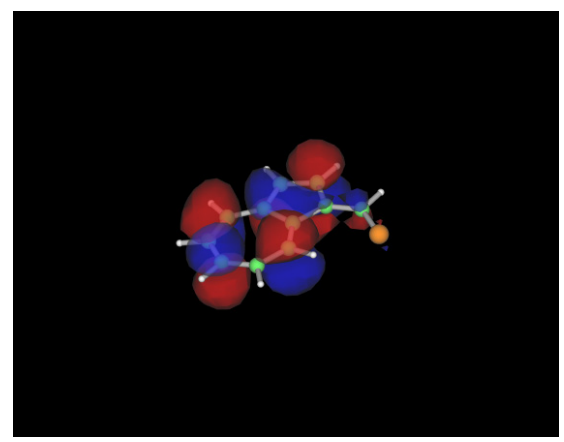

$\operatorname{HOMO}(-0.199916 \mathrm{au})$

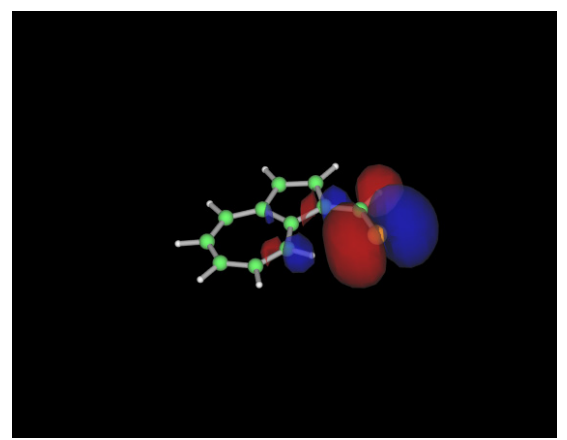

HOMO-1 (-0.207003 au)

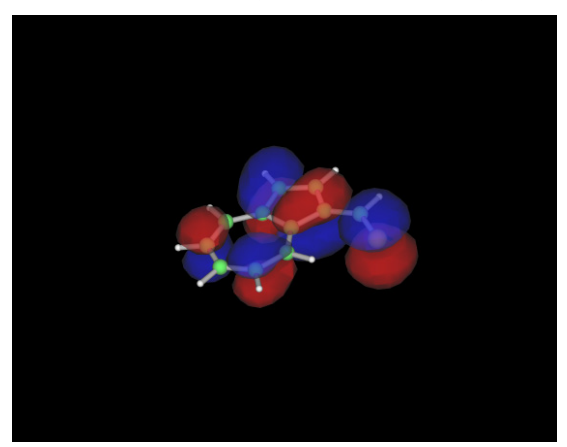

HOMO-2 (-0.251033 au)

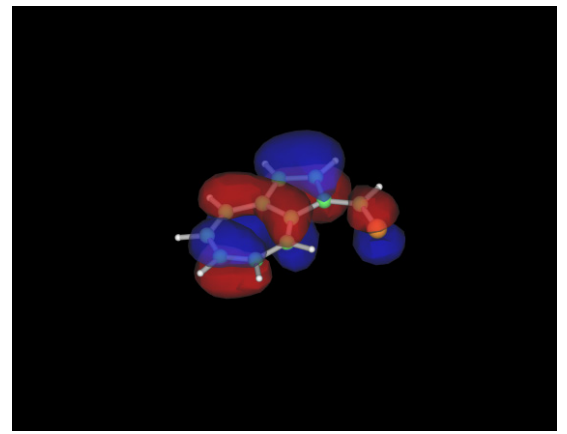

HOMO-3 (-0.290911 au)

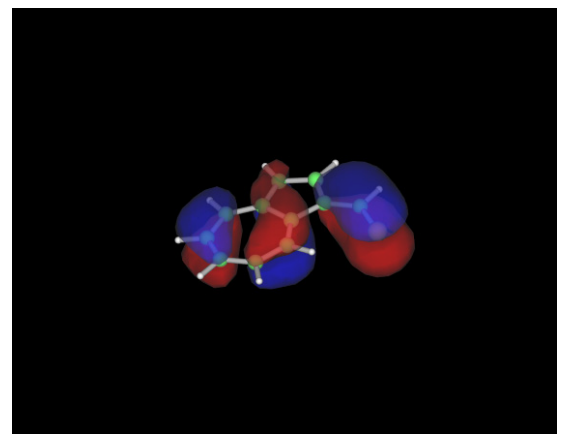

Figure S-16. Optimized structure of 1-azulenecarbothialdehyde with HOMO and LUMO orbitals calculated by B3LYP/6-31G ${ }^{* *}$ density functional theory. 


\section{Relative stability of azuleno $[8,1-b, c]$ thiophene derivatives}

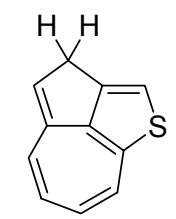

A

$0.0 \mathrm{kcal} / \mathrm{mol}$

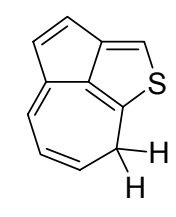

$\boldsymbol{F}$

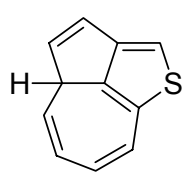

B

$11.0 \mathrm{kcal} / \mathrm{mol}$

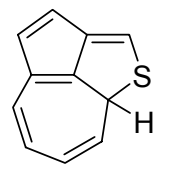

G

$17.7 \mathrm{kcal} / \mathrm{mol}$

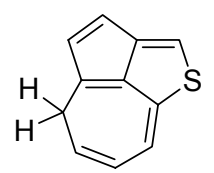

c

$16.1 \mathrm{kcal} / \mathrm{mol}$

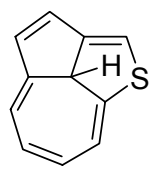

$\boldsymbol{H}$

$20.9 \mathrm{kcal} / \mathrm{mol}$

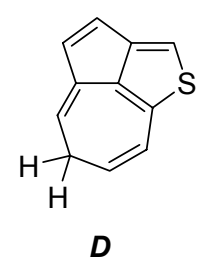

$7.9 \mathrm{kcal} / \mathrm{mol}$

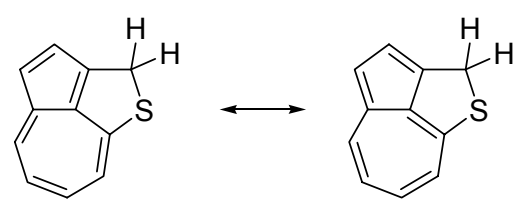

I

$-6.8 \mathrm{kcal} / \mathrm{mol}$

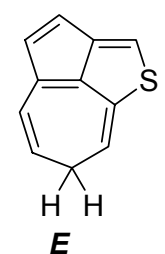

$17.7 \mathrm{kcal} / \mathrm{mol}$

$J$

$-6.9 \mathrm{kcal} / \mathrm{mol}$

Figure S-17. Relative total energy of the possible nine azuleno[8,1-b,c]thiophene derivatives with the reference to the regioisomer $\boldsymbol{A}$ calculated by the B3LYP/6-31G ${ }^{* *}$ density functional calculations. 
To estimate the relative stability during the acid-catalyzed pericyclization reaction, we have also performed the B3LYP/6-31G ${ }^{* *}$ density functional calculations of three intermediately presumed cationic species with tropylium ion structure $(\boldsymbol{A}-\boldsymbol{C})$ and the cationic structure proposed by the initial step of the acid-catalyzed pericyclization $(\boldsymbol{D})$. Consideration of the stabilities of the structural isomers, the cationic structure revealed by NMR analysis is the most stable stereoisomer among the calculated cationic species. The cationic structure proposed by the initial step of the acid-catalyzed pericyclization has rather high energy. The tropylium ion structure may be important for the stability of the intermediately presumed cationic species. The intramolecular hydrogen transfer to afford the $3 H$-azuleno[8,1-b,c]thiophene derivatives as the pericyclization products might be attributed to the energy difference.

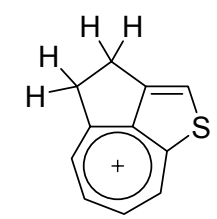

$A$

$0.0 \mathrm{kcal} / \mathrm{mol}$

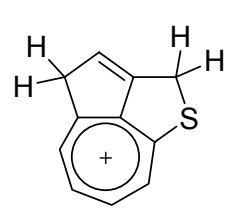

$B$

$7.3 \mathrm{kcal} / \mathrm{mol}$

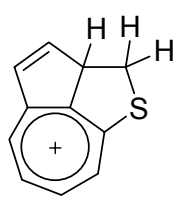

c

$14.9 \mathrm{kcal} / \mathrm{mol}$

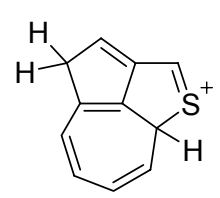

$D$

$48.6 \mathrm{kcal} / \mathrm{mol}$

Figure S-18. Relative total energy of the possible four cationic intermediates with the reference to the regioisomer $\boldsymbol{A}$ calculated by the $\mathrm{B} 3 \mathrm{LYP} / 6-31 \mathrm{G}^{* *}$ density functional calculations. 